\title{
A Bayesian analysis of generalized latent curve mixture models
}

\author{
Jun-HaO Pan*, Xin-Yuan Song ${ }^{\dagger,}$ And Edward H. IP
}

Latent curve models for longitudinal data have received increasing attention in medical, educational, psychological, and behavioral sciences. In these applied areas of research, heterogeneous longitudinal data are common. This paper proposes the use of generalized latent curve models for analyzing heterogenous longitudinal data. The basic model features a mixture of trajectories. It also employs a multinomial logit model for assessing the influence of fixed covariates and explanatory latent variables on the class membership probability within the mixture model. This broad class of models also handles non-normal data from the exponential family distributions. A Bayesian approach is implemented for data analysis. We report a simulation study that proves the satisfactory performance of the proposed approach. Furthermore, we analyzed a real data set extracted from the National Longitudinal Survey of Youth to illustrate the practical value of the proposed model and methodology.

KEYWORDS AND PHRASES: Latent curve mixture models, Heterogeneous longitudinal data, Markov chain Monte Carlo method, Modified deviance information criterion.

\section{INTRODUCTION}

Longitudinal data, which comprise repeated measurements of the same individuals on different periods, arise frequently in a wide range of fields, such as psychology, education, medicine, and public health. Latent curve modeling (LCM) $[3,23,24$, among others], which applies structural equation modeling (SEM) concepts and techniques to traditional growth curve analyses, has received increasing attention as a useful longitudinal technique in the analysis of change patterns. LCM can incorporate information about group and individuals. More importantly, LCM can be used to analyze and explain changes. For example, LCM relates

*Jun-Hao Pan's research is fully supported by grants from the National Natural Science Foundation of China (NSFC 11026192 and NSFC 91124004). This research is also supported by the Fundamental Research Funds for the Central Universities and the high-performance grid computing platform of Sun Yat-sen University.

†Prof. Xin-Yuan Song's research is fully supported by grants (GRF 446609 and GRF 404711) from the Research Grant Council of the Hong Kong Special Administration.

${ }^{\ddagger}$ Corresponding author. growth factors to an individual's contextual data by extracting latent growth factors from individual trajectories. Based on the pioneer work of Meredith and Tisak [23, 24], several scholars demonstrated that LCM is a useful tool for studying developmental trends from both inter- and intra-individual perspectives [see, for example, 3, 8, 22, 26, 27, 29, 37, 41, among others].

Heterogeneity is an important issue in the analysis of longitudinal data. Statistical inference would be seriously distorted when a heterogeneous population is analyzed as homogeneous. Observed heterogeneity can be captured by fixed-effects models, including multi-group models (e.g., age groups). However, other types of heterogeneities such as heterogeneous trajectory classes cannot be handled by fixedeffects models because an individual's membership in a trajectory class is unknown in advance. In statistics, a discrete or continuous mixing distribution is often used to model heterogeneity. When a discrete mixing distribution is deployed, the model is often termed a finite mixture model. In longitudinal studies, the usefulness of mixture models has been increasingly recognized for identifying meaningful classes of individuals according to their developmental trends. Recently, Muthén et al. [see, for example, 26, 28, among others] extended the classical LCM to finite-mixture LCMs and proposed a growth mixture modeling (GMM) under which a population of interest is regarded as a mixture of classes, each defined by its distinct developmental trajectory. In their models, probabilities of class membership can be directly related to covariates through a multinomial logit model. Hence, the model for membership belonging to a specific trajectory class is enhanced with the information derived from the covariates. For instance, if male students are more likely to belong to a trajectory class of faster learners in mathematics, then the predictive power of the mixture model can be improved by including gender as a covariate.

There are two significant methodological challenges for applying GMMs to real data analysis, especially in the social and behavioral sciences. First, explanatory variables are often measured indirectly. Thus, the concept of a latent explanatory variable is useful when multiple indicators are used to describe a specific construct. Second, in practice, the makeup of multiple indicators for a specific construct is almost always multimodal. For example, binary and continuous (including normal and non-normal distributions) data could be present. Sometimes, ad hoc methods are used to 
create single-mode data. For example, cutoffs are set for continuous data. However, direct models for mixed-mode data would greatly increase the flexibility of the analytic tool. To the best of our knowledge, limited work has been conducted to fully address these practical issues within the context of GMMs.

To illustrate the aforementioned statistical challenges, we used an example from the National Longitudinal Surveys of Youth (NLSY79) in the U.S. This example was also used to illustrate our proposed solution. NLSY79 is a nationally representative sample of young men and women 14 to 22 years of age at the time of the first survey in 1979. These individuals were interviewed annually until 1994 and are currently interviewed on a biennial basis. Their scores for the Peabody Individual Achievement Tests (PIATs) [10] in mathematics and other subjects were recorded at each period. The data analysis was conducted to investigate the longitudinal behaviors of the mathematics achievement of young students from 1990 to 1994 and to identify whether different classes of developmental trends exist. The effects of some important determinants on academic achievement were also determined. Unlike gender, which is directly observed, other explanatory variables of growth trajectory such as behavior problems and home environment are indirectly measured using the five indicators of the Behavior Problem Index (BPI) [43] and the three indicators of the Home Observation for Measurement of the Environment (HOME) Inventory [5]. The indicators of these latent constructs do not necessarily belong to a single data type. For example, the latent explanatory variable "home environment" is described by binary variables such as "live with mother" and by continuous variables such as "cognitive stimuli score". Although this example concerns a social survey, the two challengesexplanatory latent variables and mixed-mode indicatorsare common in other sciences, including health and business/economics.

In this paper, we propose a generalized latent curve mixture model (GLCMM) to analyze heterogeneous longitudinal data. The proposed approach directly addresses the aforementioned statistical challenges. By building upon existing work in GMM, our proposed model aims to fill the following significant gaps in the literature by (1) incorporating explanatory latent variables for predicting latent growth factors and probability of class membership, and by (2) including a broad class of data types for indicators of the latent variables through the exponential family distribution (EFD) framework. We adopted a Bayesian approach, together with Markov chain Monte Carlo (MCMC) techniques such as the Gibbs sampler [15], the Metropolis-Hastings (MH) algorithm [17, 25], and the permutation sampling [14], to analyze GLCMM. A Bayesian model selection criterion, the modified deviance information criterion (DIC), was utilized for model selection. The sampling-based Bayesian approach is proposed for several reasons. First, the different types of latent variables (categorical and continuous) and complicated data structure make the statistical inference of GLCMM through the maximum likelihood (ML)-based method difficult. By contrast, the sampling-based Bayesian approach is powerful for analyzing complex models and data. Second, the ML-based method is likely to encounter multiple modes in a mixture likelihood, which could lead to uncertainty in inference if all the modes are reached [12]. Our proposed Bayesian approach employs permutation sampling to fix the problem of label switching in a mixture likelihood and to avoid getting trapped in a suboptimal mode. Finally, the Bayesian approach allows the use of genuine prior information and is less dependent on asymptotic theory, thereby producing more reliable results even with small samples $[11$, 19, 32, 37, among others].

The remainder of this paper is organized as follows. Section 2 describes GLCMM. Section 3 presents a Bayesian analysis of the proposed model. An MCMC method coupled with the permutation sampling is proposed to obtain the Bayesian estimates of the unknown parameters. The modified DIC is used for model selection. Section 4 shows a simulation study for the evaluation of the empirical performance of Bayesian estimation and model selection. Section 5 illustrates the methodology with a real data set from NLSY79. Section 6 concludes the paper with a discussion.

\section{GENERALIZED LATENT CURVE MIXTURE MODEL (GLCMM)}

\subsection{Basic latent curve model}

LCMs are popular longitudinal techniques for analyzing individual differences in changing patterns, which usually involve random intercepts and slopes, with each pair forming a different trajectory over time. The basic LCM can be viewed as the following common factor analysis model or trajectory equation by Bollen and Curran [3]:

$$
\mathbf{y}_{i}=\boldsymbol{\Lambda}_{y} \boldsymbol{\eta}_{i}+\boldsymbol{\epsilon}_{y i}, \quad i=1, \ldots, n
$$

where $\mathbf{y}_{i}=\left(y_{i 1}, y_{i 2}, \ldots, y_{i, p_{1}}\right)^{T}$ is a $p_{1} \times 1$ vector of continuous repeated measures at $p_{1}$ periods for the $i$-th individual, $\boldsymbol{\Lambda}_{y}$ is a $p_{1} \times q_{1}$ matrix of sequentially known values of the growth curve records, $\boldsymbol{\eta}_{i}$ is a $q_{1} \times 1$ latent growth factor, and $\boldsymbol{\epsilon}_{y i}$ is a $p_{1} \times 1$ vector of error measurements, which is assumed to be independent of $\boldsymbol{\eta}_{i}$ and distributed as $N\left(\mathbf{0}, \boldsymbol{\Psi}_{y}\right)$ with a diagonal covariance matrix $\boldsymbol{\Psi}_{y}$. The pattern of $\boldsymbol{\Lambda}_{y}$ can be interpreted as a representation of a particular aspect of change in $y_{i j}$ across $p_{1}$ periods. When $q_{1}=2$, Equation (1) for a linear LCM is expressed in the following matrix form:

$$
\left(\begin{array}{c}
y_{i 1} \\
y_{i 2} \\
\vdots \\
y_{i, p_{1}}
\end{array}\right)=\left(\begin{array}{cc}
1 & t_{1} \\
1 & t_{2} \\
\vdots & \vdots \\
1 & t_{p_{1}}
\end{array}\right)\left(\begin{array}{l}
\eta_{i 1} \\
\eta_{i 2}
\end{array}\right)+\left(\begin{array}{c}
\epsilon_{y i 1} \\
\epsilon_{y i 2} \\
\vdots \\
\epsilon_{y i, p_{1}}
\end{array}\right)
$$


The first column of $\boldsymbol{\Lambda}_{y}$ is used to define an intercept factor, which represents the initial status of change in $y_{i j}$. The second column of $\boldsymbol{\Lambda}_{y}$ represents known times of measurement. The values of $t_{j}\left(j=1, \ldots, p_{1}\right)$ reflect the spacing between measurement periods, and the latent growth factor $\boldsymbol{\eta}_{i}$ contains the random intercept (initial status) $\eta_{i 1}$ and random slope (rate of change) $\eta_{i 2}$. The two-factor LCM in this case is specified so that the intercept factor serves as the starting point for any growth across time and the slope factor captures the rate of change in the trajectory over time. The values of $t_{j}\left(j=1, \ldots, p_{1}\right)$ can be specified using either fixed value restrictions (e.g. $0,1, \ldots, p_{1}-1$ ) that represent a straight-line growth or unspecified value restrictions $\left(t_{1}=0\right.$ and $t_{2}=1$ are fixed for model identification, and the remaining $t_{3}, \ldots, t_{p_{1}}$ are freely estimated) that allow estimation of an optimal pattern of change over measurement periods [24]. However, the method of unspecified value restrictions does not produce a straightforward interpretation of the resulting model parameters; see [3] for more details. In this article, we only consider the fixed value restrictions method.

To examine variabilities across individuals, the latent growth factor $\boldsymbol{\eta}_{i}$ is further modeled by

$$
\boldsymbol{\eta}_{i}=\boldsymbol{\mu}+\boldsymbol{\delta}_{i}, \quad i=1,2, \ldots, n,
$$

where $\boldsymbol{\mu}$ is a $q_{1} \times 1$ vector of the population mean of latent individual growth factors, $\boldsymbol{\delta}_{i}$ is a $q_{1} \times 1$ random vector of residuals that reflect differences between the mean growth factors and the individual growth factors, and $\boldsymbol{\delta}_{i}$ is assumed to be distributed as $N\left(\mathbf{0}, \Psi_{\delta}\right)$ with a diagonal covariance matrix $\boldsymbol{\Psi}_{\delta}$. The diagonal elements $\psi_{\delta j}$ measure the degree of diversity in individual latent growth factors from their means.

For instance, in the study of NLSY79 described in Section 1 for investigating the longitudinal behavior of mathematics achievement of young students, we aim to specify an LCM to represent changes in math scores. To obtain a rough idea about the trajectory model, we plotted the average PIAT math scores across all individuals at different periods. Figure 1 shows that a linear trajectory model is adequate. We denote $y_{i j}(j=1,2,3)$ as the PIAT scores [10] in mathematics in 1990, 1992, and 1994, during which the measurements were conducted. A linear LCM for $\mathbf{y}_{i}=\left(y_{i 1}, y_{i 2}, y_{i 3}\right)^{T}$ is

$$
\begin{aligned}
& \left(\begin{array}{l}
y_{i 1} \\
y_{i 2} \\
y_{i 3}
\end{array}\right)=\left(\begin{array}{ll}
1 & 0 \\
1 & 1 \\
1 & 2
\end{array}\right)\left(\begin{array}{l}
\eta_{i 1} \\
\eta_{i 2}
\end{array}\right)+\left(\begin{array}{l}
\epsilon_{y i 1} \\
\epsilon_{y i 2} \\
\epsilon_{y i 3}
\end{array}\right), \\
& \left(\begin{array}{l}
\eta_{i 1} \\
\eta_{i 2}
\end{array}\right)=\left(\begin{array}{l}
\mu_{1} \\
\mu_{2}
\end{array}\right)+\left(\begin{array}{l}
\delta_{i 1} \\
\delta_{i 2}
\end{array}\right),
\end{aligned}
$$

where the known times of measurement are set to 0,1 , and 2 to reflect the equal spacing between measurement periods from 1990 to 1994, and the subject developmental trend of

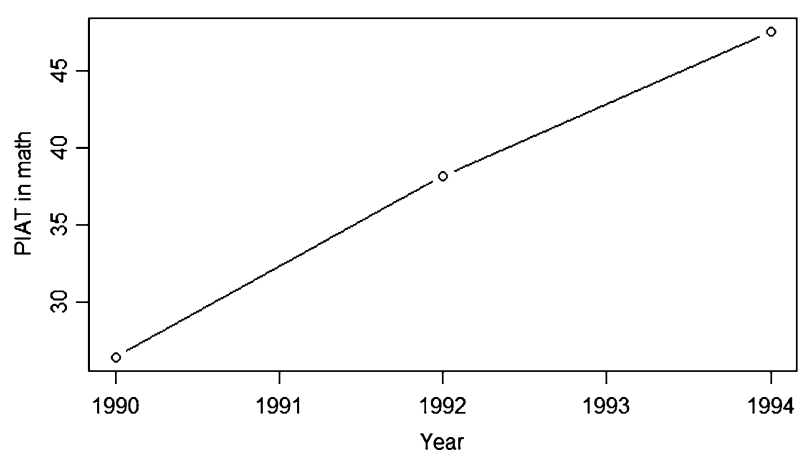

Figure 1. Trajectory of average PIAT math scores across all individuals at three measurement periods.

mathematics score is described by a linear trajectory with the random intercept $\eta_{i 1}$ and random slope $\eta_{i 2}$.

In some circumstances, a linear LCM may not be flexible enough to model practical situations. The use of a polynomial would help capture a nonlinear trajectory. For instance, a quadratic LCM is defined by

$$
\begin{aligned}
\left(\begin{array}{c}
y_{i 1} \\
y_{i 2} \\
\vdots \\
y_{i, p_{1}}
\end{array}\right) & =\left(\begin{array}{ccc}
1 & 0 & 0^{2} \\
1 & 1 & 1^{2} \\
\vdots & \vdots & \vdots \\
1 & p_{1}-1 & \left(p_{1}-1\right)^{2}
\end{array}\right)\left(\begin{array}{l}
\eta_{i 1} \\
\eta_{i 2} \\
\eta_{i 3}
\end{array}\right)+\left(\begin{array}{c}
\epsilon_{y i 1} \\
\epsilon_{y i 2} \\
\vdots \\
\epsilon_{y i, p_{1}}
\end{array}\right), \\
\left(\begin{array}{l}
\eta_{i 1} \\
\eta_{i 2} \\
\eta_{i 3}
\end{array}\right) & =\left(\begin{array}{l}
\mu_{1} \\
\mu_{2} \\
\mu_{3}
\end{array}\right)+\left(\begin{array}{c}
\delta_{i 1} \\
\delta_{i 2} \\
\delta_{i 3}
\end{array}\right),
\end{aligned}
$$

where $\eta_{i 3}$ is the quadratic component for revealing the curvature presented in individual trajectories. In practice, a model selection procedure may be conducted to determine whether the inclusion of additional nonlinear terms leads to a significant improvement in model fit.

\subsection{Generalized latent curve model}

In substantive research, the longitudinal patterns of individuals may be influenced by other observed and latent explanatory variables. Moreover, the latent growth factors in $\boldsymbol{\eta}_{i}$ may be inter-correlated. To assess these effects and inter-relationships, Equation (2) in the basic LCM can be extended to

$$
\boldsymbol{\eta}_{i}=\boldsymbol{\mu}+\mathbf{A} \mathbf{w}_{i}+\boldsymbol{\Pi} \boldsymbol{\eta}_{i}+\boldsymbol{\Gamma} \mathbf{F}\left(\boldsymbol{\xi}_{i}\right)+\boldsymbol{\delta}_{i},
$$

where $\boldsymbol{\mu}$ is a $q_{1} \times 1$ vector of intercepts, $\mathbf{w}_{i}$ is an $r_{1} \times 1$ vector of fixed covariates that may follow either continuous or discrete distributions, and $\boldsymbol{\xi}_{i}$ is a $q_{2} \times 1$ vector of explanatory latent variables. $\mathbf{F}\left(\boldsymbol{\xi}_{i}\right)=\left(f_{1}\left(\boldsymbol{\xi}_{i}\right), \ldots, f_{h}\left(\boldsymbol{\xi}_{i}\right)\right)^{T}$ is an $h \times 1$ vector-valued function that contains nonzero differentiable functions $f_{1}, \ldots, f_{h}$, the forms of which are given and may need to be tested. Furthermore, $\mathbf{A}$ and $\boldsymbol{\Gamma}$ are $q_{1} \times r_{1}$ and $q_{1} \times h$ matrices of unknown parameters reflecting the effects 
of $\mathbf{w}_{i}$ and $\mathbf{F}\left(\boldsymbol{\xi}_{i}\right)$ on $\boldsymbol{\eta}_{i}$, and $\boldsymbol{\Pi}$ is a $q_{1} \times q_{1}$ matrix of unknown coefficients specifying the inter-relationships among the latent growth factors in $\boldsymbol{\eta}_{i}$. We assume that the distribution of $\boldsymbol{\delta}_{i}$ is $N\left(\mathbf{0}, \Psi_{\delta}\right)$ with a diagonal covariance matrix $\boldsymbol{\Psi}_{\delta}$ and that $\boldsymbol{\delta}_{i}$ is independent of $\boldsymbol{\xi}_{i}$. Let $\boldsymbol{\Pi}_{0}=\mathbf{I}-\boldsymbol{\Pi}$, in which $\boldsymbol{\Pi}_{0}$ is assumed to be non-singular and $\left|\boldsymbol{\Pi}_{0}\right|$ is assumed to be independent of the elements of $\boldsymbol{\Pi}$. Li and Wang [21] reported that the violation of this assumption would cause a nonstandard and complex full conditional distribution of $\boldsymbol{\Pi}$, thereby requiring a tedious step for drawing observations from $p(\boldsymbol{\Pi} \mid \cdot)$ in the MCMC algorithm. In this article, we assume that $\left|\boldsymbol{\Pi}_{0}\right|$ is a constant independent of $\boldsymbol{\Pi}$ for the sake of simple computation. This assumption can be relaxed with a modification of the posterior sampling [21].

In the NLSY79 study, how "behavior problems" influence the developmental trend of PIAT mathematics score is of particular interest. However, "behavior problems, $\xi_{i}$ " is a latent variable because it is measured by the multiple items of BPI [43]. A model incorporating the effects of $\xi_{i}$ on the latent growth factors is

$$
\left(\begin{array}{l}
\eta_{i 1} \\
\eta_{i 2}
\end{array}\right)=\left(\begin{array}{l}
\mu_{1} \\
\mu_{2}
\end{array}\right)+\left(\begin{array}{l}
\gamma_{1} \\
\gamma_{2}
\end{array}\right) \xi_{i}+\left(\begin{array}{c}
\delta_{i 1} \\
\delta_{i 2}
\end{array}\right)
$$

which is a special case of Equation (5) with $\mathbf{A}=\mathbf{0}, \boldsymbol{\Pi}=$ $\mathbf{0}$, and $\mathbf{F}\left(\boldsymbol{\xi}_{i}\right)=\xi_{i}$. The regression coefficients $\gamma_{1}$ and $\gamma_{2}$ represent the effect of behavior problems on the initial status and rate of change in mathematics score, respectively.

The latent vector $\boldsymbol{\xi}_{i}$ in Equation (5) can be measured as follows:

$$
\mathbf{x}_{i}=\boldsymbol{\Lambda}_{x} \boldsymbol{\xi}_{i}+\boldsymbol{\epsilon}_{x i}, \quad i=1,2, \ldots, n
$$

where $\mathbf{x}_{i}$ is a $p_{2} \times 1$ vector of observed indicators, $\boldsymbol{\Lambda}_{x}$ is a $p_{2} \times q_{2}$ factor loading matrix, and $\boldsymbol{\epsilon}_{x i}$ is a $p_{2} \times 1$ random vector of error measurements. Here, $\boldsymbol{\epsilon}_{x i}$ is assumed to be independent of $\boldsymbol{\xi}_{i}, \boldsymbol{\eta}_{i}, \boldsymbol{\epsilon}_{y i}$, and $\boldsymbol{\delta}_{i}$, and distributed as $N\left(\mathbf{0}, \boldsymbol{\Psi}_{x}\right)$ with diagonal $\boldsymbol{\Psi}_{x}$, and $\boldsymbol{\xi}_{i}$ is assumed to be distributed as $N(\mathbf{0}, \boldsymbol{\Phi})$. Note that Models (1) and (7) are confirmatory factor analysis (CFA) models because the patterns of their factor loading matrices can be pre-specified according to background data.

In the study of NLSY79 data, "behavior problems, $\xi_{i}$ " is measured by the indicators, $x_{i 1}, x_{i 2}, x_{i 3}, x_{i 4}$, and $x_{i 5}$, which are the BPI [43] subscales for antisocial, anxious, peer conflict, headstrong, and hyperactive behaviors, respectively. Equation (7) is then defined with $p_{2}=5$ and $q_{2}=1$ :

$$
\left(\begin{array}{l}
x_{i 1} \\
x_{i 2} \\
x_{i 3} \\
x_{i 4} \\
x_{i 5}
\end{array}\right)=\left(\begin{array}{c}
1.0 \\
\lambda_{x 21} \\
\lambda_{x 31} \\
\lambda_{x 41} \\
\lambda_{x 51}
\end{array}\right) \xi_{i}+\left(\begin{array}{c}
\epsilon_{x i 1} \\
\epsilon_{x i 2} \\
\epsilon_{x i 3} \\
\epsilon_{x i 4} \\
\epsilon_{x i 5}
\end{array}\right)
$$

where 1.0 is fixed to identify the model and to introduce a scale to the latent variable $\xi_{i}$. Figure 2 depicts the path diagram of LCM for analyzing the NLSY79 data.

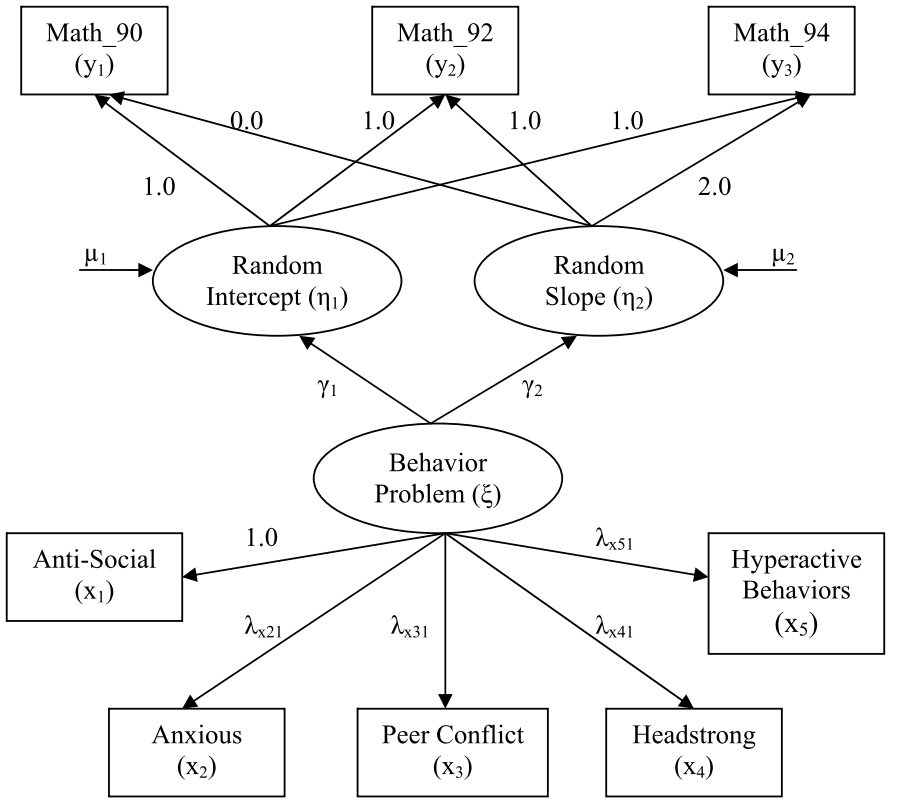

Figure 2. Path diagram of the generalized linear LCM for analyzing the NLSY79 data.

Although we only cope with the linear effect of $\xi_{i}$ on $\boldsymbol{\eta}_{i}$ in Figure 2, Equation (5) is flexible enough to assess the effects of the observed covariates in $\mathbf{w}_{i}$ on $\boldsymbol{\eta}_{i}$, the interrelationships among the growth factors in $\boldsymbol{\eta}_{i}$, and the nonlinear effect of $\boldsymbol{\xi}_{i}$ on $\boldsymbol{\eta}_{i}$. For example,

$$
\begin{aligned}
\left(\begin{array}{l}
\eta_{i 1} \\
\eta_{i 2}
\end{array}\right)= & \left(\begin{array}{l}
\mu_{1} \\
\mu_{2}
\end{array}\right)+\left(\begin{array}{ll}
a_{11} & a_{12} \\
a_{21} & a_{22}
\end{array}\right)\left(\begin{array}{l}
w_{1} \\
w_{2}
\end{array}\right)+\left(\begin{array}{cc}
0 & 0 \\
\pi_{21} & 0
\end{array}\right)\left(\begin{array}{l}
\eta_{i 1} \\
\eta_{i 2}
\end{array}\right) \\
& +\left(\begin{array}{lll}
\gamma_{11} & \gamma_{12} & \gamma_{13} \\
\gamma_{21} & \gamma_{22} & \gamma_{23}
\end{array}\right)\left(\begin{array}{c}
\xi_{i 1} \\
\xi_{i 2} \\
\xi_{i 1} \xi_{i 2}
\end{array}\right)+\left(\begin{array}{l}
\delta_{i 1} \\
\delta_{i 2}
\end{array}\right)
\end{aligned}
$$

where $\pi_{21}$ reveals the interrelationship between $\eta_{i 1}$ and $\eta_{i 2}$, and $\gamma_{13}$ and $\gamma_{23}$ examines the interaction effects of $\xi_{i 1}$ and $\xi_{i 2}$ on $\eta_{i 1}$ and $\eta_{i 2}$, respectively. The incorporation of nonlinear $\mathbf{F}\left(\boldsymbol{\xi}_{i}\right)$ in Equation (5) is important to provide a general model that reflects the true relationships among latent variables. The distribution of $\mathbf{y}_{i}$ is non-normal because of the nonlinearity of $\mathbf{F}\left(\boldsymbol{\xi}_{i}\right)$. Hence, the conventional computer packages developed based on the normal assumption of $\mathbf{y}_{i}$ cannot be directly applied to the current analysis.

As mentioned above, in Equation (5), $\left|\boldsymbol{\Pi}_{0}\right|=|\mathbf{I}-\boldsymbol{\Pi}|$ is independent of the elements of $\boldsymbol{\Pi}$. In some circumstances, this assumption may be violated. For example,

$$
\left(\begin{array}{l}
\eta_{i 1} \\
\eta_{i 2}
\end{array}\right)=\left(\begin{array}{l}
\mu_{1} \\
\mu_{2}
\end{array}\right)+\left(\begin{array}{cc}
0 & \pi_{12} \\
\pi_{21} & 0
\end{array}\right)\left(\begin{array}{l}
\eta_{i 1} \\
\eta_{i 2}
\end{array}\right)+\left(\begin{array}{c}
\delta_{i 1} \\
\delta_{i 2}
\end{array}\right),
$$

where $\left|\boldsymbol{\Pi}_{0}\right|=|\mathbf{I}-\boldsymbol{\Pi}|=1-\pi_{12} \pi_{21}$. The nonconstant $\left|\mathbf{\Pi}_{0}\right|$ causes a nonstandard posterior distribution of $p(\boldsymbol{\Pi} \mid \cdot)$. The MCMC method developed by Li and Wang [21] can be applied to sampling from $p(\mathbf{\Pi} \mid \cdot)$. 
The generalized LCMs defined by Equations (1), (5), and (7) are not identified without imposing appropriate identification constraints. For instance, an equivalent form of (7) is $\mathbf{x}_{i}=\boldsymbol{\Lambda}_{x}^{*} \boldsymbol{\xi}_{i}^{*}+\boldsymbol{\epsilon}_{x i}$, where $\boldsymbol{\Lambda}_{x}^{*}=\boldsymbol{\Lambda}_{x} \mathbf{R}, \boldsymbol{\xi}_{i}^{*}=\mathbf{R}^{-1} \boldsymbol{\xi}_{i}$, and $\mathbf{R}$ is an arbitrary nonsingular matrix. One common method of solving this problem is to fix appropriate elements in $\boldsymbol{\Lambda}_{x}$ at fixed known values so that the only possible choice of $\mathbf{R}$ is the identity matrix $[2,18,33]$. In Equation (8), for example, we fix $\lambda_{x 11}$ at 1.0 for the purpose of identification. Similarly, appropriate elements in $\boldsymbol{\Lambda}_{y}, \boldsymbol{\Pi}$, and/or $\boldsymbol{\Gamma}$ may also be fixed at known values if necessary.

CFA models (1) and (7) can be synthesized into a unique model framework. Assuming that $\mathbf{u}_{i}=\left(\mathbf{y}_{i}^{T}, \mathbf{x}_{i}^{T}\right)^{T}, \boldsymbol{\Lambda}=$ $\left(\begin{array}{cc}\boldsymbol{\Lambda}_{y} & \mathbf{0} \\ \mathbf{0} & \boldsymbol{\Lambda}_{x}\end{array}\right), \boldsymbol{\omega}_{i}=\left(\boldsymbol{\eta}_{i}^{T}, \boldsymbol{\xi}_{i}^{T}\right)^{T}$, and $\boldsymbol{\epsilon}_{u i}=\left(\boldsymbol{\epsilon}_{y i}^{T}, \boldsymbol{\epsilon}_{x i}^{T}\right)^{T}$, then (1) and (7) can be combined as

$$
\mathbf{u}_{i}=\boldsymbol{\Lambda} \boldsymbol{\omega}_{i}+\boldsymbol{\epsilon}_{u i}, \quad i=1,2, \ldots, n,
$$

where $\mathbf{u}_{i}(p \times 1)$ consists of $p_{1}$ repeated measurements in (1) and $p_{2}$ multiple indicators in (7), $\boldsymbol{\omega}_{i}(q \times 1)$ consists of $q_{1}$ latent growth factors in $\boldsymbol{\eta}_{i}$ and $q_{2}$ explanatory latent variables in $\boldsymbol{\xi}_{i}$, where $p=p_{1}+p_{2}$, and $q=q_{1}+q_{2}$. With this synthesis, the generalized LCM can be simply defined by Equations (5) and (9).

\subsection{Generalized latent curve mixture model}

To accommodate possible heterogeneity in longitudinal data, we further introduce a latent allocation variable to the model described in Section 2.2. The model consists of two parts. The first part (the upper panel of Figure 3) defines a mixture model with latent trajectory classes, in which the probability of an individual belonging to a trajectory class is predicted by explanatory latent variables in $\zeta$ and observed covariates in $\mathbf{b}$ through a multinomial logit model. The second part (the lower panel of Figure 3) is a withinclass LCM that examines how explanatory latent variables in $\boldsymbol{\xi}$ and observed covariates in $\mathbf{w}$ affect latent growth factors in $\boldsymbol{\eta}$ within a trajectory class. Each part is presented in the following sections.

\subsubsection{Mixture modeling for latent trajectory classes}

Suppose that $K$ different trajectory classes are present in a random sample of size $n$, where $K$ is the number of trajectory classes and is usually determined based on a model selection procedure. A mixture model for $\mathbf{u}_{i}$ is defined as follows:

$$
p\left(\mathbf{u}_{i}\right)=\sum_{k=1}^{K} \pi_{i k} p_{k}\left(\mathbf{u}_{i} \mid \boldsymbol{\theta}_{k}\right), \quad i=1,2, \ldots, n,
$$

where $\pi_{i k}$ is the probability of the $i$ th individual in the $k$ th class such that $\sum_{k=1}^{K} \pi_{i k}=1.0$, and $p_{k}(\cdot \mid \cdot)$ is the density function of $\mathbf{u}_{i}$ with parameter vector $\boldsymbol{\theta}_{k}$. The probability $\pi_{i k}$ can be further modeled by the multinomial logit model:

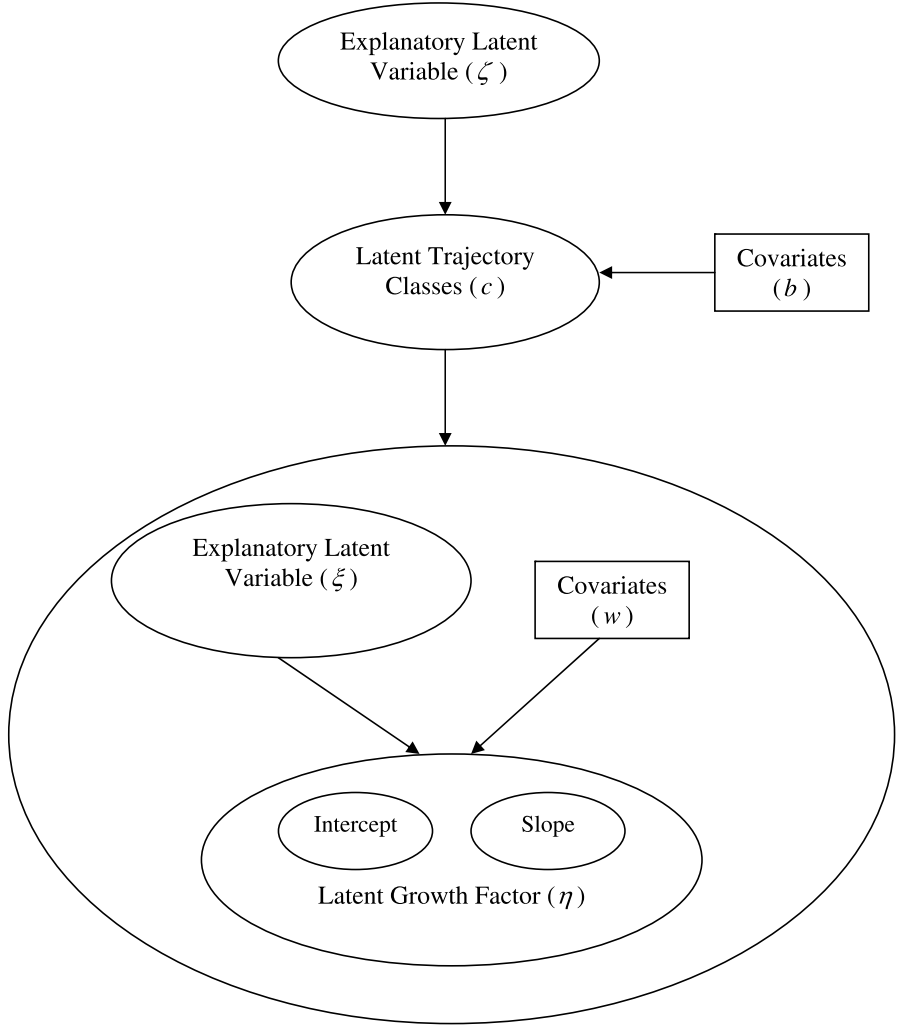

Figure 3. Structure of GLCMM.

$$
\begin{aligned}
\pi_{i k} & =p\left(c_{i}=k \mid \mathbf{b}_{i}, \boldsymbol{\zeta}_{i}\right) \\
& =\frac{\exp \left(\varphi_{k 0}+\boldsymbol{\varphi}_{k}^{(1)^{T}} \mathbf{b}_{i}+\boldsymbol{\varphi}_{k}^{(2)^{T}} \boldsymbol{\zeta}_{i}\right)}{\sum_{l=1}^{K} \exp \left(\varphi_{l 0}+\boldsymbol{\varphi}_{l}^{(1)^{T}} \mathbf{b}_{i}+\boldsymbol{\varphi}_{l}^{(2)^{T}} \boldsymbol{\zeta}_{i}\right)}
\end{aligned}
$$

where $c_{i}$ is a latent allocation variable, $\mathbf{b}_{i}$ is an $r_{2} \times 1$ vector of fixed covariates, $\boldsymbol{\zeta}_{i}$ is a $q_{3} \times 1$ vector of explanatory latent variables, and $\varphi_{k 0}, \varphi_{k}^{(1)}=\left(\varphi_{k 1}, \varphi_{k 2}, \ldots, \varphi_{k, r_{2}}\right)^{T}$, and $\varphi_{k}^{(2)}=$ $\left(\varphi_{k, r_{2}+1}, \varphi_{k, r_{2}+2}, \ldots, \varphi_{k, r_{2}+q_{3}}\right)^{T}$ are class-specific unknown parameters. To identify (11), $\varphi_{K 0}, \varphi_{K 1}, \ldots, \varphi_{K, r_{2}+q_{3}}$ are fixed at 0.0. The explanatory latent vector $\boldsymbol{\zeta}_{i}$ in (11) is measured by multiple indicators:

$$
\mathbf{v}_{i}=\boldsymbol{\Lambda}_{v} \boldsymbol{\zeta}_{i}+\boldsymbol{\epsilon}_{v i}, \quad i=1,2, \ldots, n,
$$

where $\mathbf{v}_{i}$ is a $p_{3} \times 1$ vector of observed indicators, $\boldsymbol{\Lambda}_{v}$ is a $p_{3} \times$ $q_{3}$ factor loading matrix, and $\boldsymbol{\epsilon}_{v i}$ is a $p_{3} \times 1$ vector of random errors with distribution $N\left(\mathbf{0}, \Psi_{v}\right)$, where $\boldsymbol{\Psi}_{v}$ is diagonal. In the study of the NSLY79 data, $p_{3}=3$ and $q_{3}=1$. The observed indicators are household variable, home cognitive stimulation score, and emotional support score, which are used to measure the latent variable "home environment". We assume that $\boldsymbol{\zeta}_{i}$ is independent of $\boldsymbol{\epsilon}_{v i}$ and distributed as $N\left(\mathbf{0}, \boldsymbol{\Phi}_{v}\right)$, where $\boldsymbol{\Phi}_{v}$ is an unknown covariance matrix. Model (12) can be identified by fixing appropriate elements in $\boldsymbol{\Lambda}_{v}$ at known values. 
Compared with the conventional multinomial logit model, Model (11) involves fixed covariates and latent variables as predictors. The main goal of this extension is to provide a more flexible model for better prediction of the unknown probability of class membership. In Equations (5) and (11), and (7) and (12), common variables between $\mathbf{w}_{i}$ and $\mathbf{b}_{i}, \mathbf{v}_{i}$ and $\mathbf{x}_{i}$, and $\boldsymbol{\xi}_{i}$ and $\boldsymbol{\zeta}_{i}$ may exist, respectively.

In the study of the NLSY79 data, "home environment" and "gender" are of interest in predicting the probability of class membership. Model (11) with $p_{3}=3$ and $q_{3}=r_{2}=1$ can be written as

(13)

$$
\pi_{i k}=p\left(c_{i}=k \mid b_{i}, \zeta_{i}\right)=\frac{\exp \left(\varphi_{k 0}+\varphi_{k 1} b_{i}+\varphi_{k 2} \zeta_{i}\right)}{\sum_{l=1}^{K} \exp \left(\varphi_{l 0}+\varphi_{l 1} b_{i}+\varphi_{l 2} \zeta_{i}\right)},
$$

where $b_{i}$ indicates the observed covariate "gender", and $\zeta_{i}$ indicates the latent variable "home environment", which is measured by the indicators of household variable, home cognitive stimulation score, and emotional support score via Equation (12).

\subsubsection{Within-class generalized latent curve model}

In Section 2.3.1, we defined a mixture model, in which $\mathbf{u}_{i}$ is assumed to come from $K$ latent trajectory classes. In this section, we further define the within-class model. Given that $c_{i}=k, k=1, \ldots, K$, the within-class generalized LCM can be defined as:

$$
\begin{aligned}
& \mathbf{u}_{i}=\boldsymbol{\Lambda}_{k} \boldsymbol{\omega}_{i}+\boldsymbol{\epsilon}_{u i}, \quad i=1,2, \ldots, n, \\
& \boldsymbol{\eta}_{i}=\boldsymbol{\mu}_{k}+\mathbf{A}_{k} \mathbf{w}_{i}+\boldsymbol{\Pi}_{k} \boldsymbol{\eta}_{i}+\boldsymbol{\Gamma}_{k} \mathbf{F}\left(\boldsymbol{\xi}_{i}\right)+\boldsymbol{\delta}_{i},
\end{aligned}
$$

where all the components are defined in a similar manner as those in Equations (5) and (9) except that the unknown parameters are class-specific. The models defined in Equations (14) and (15) allow for heterogeneity both in the patterns of trajectories and in the effects of explanatory latent and observed variables on the latent growth factors. These models are characterized by a form of invariance [42], that is, the fixed parameters are fixed at the same locations in different trajectory classes. For instance, in Equation (8), we fix the element $\lambda_{x 11}$ at 1.0 to identify the model in each trajectory class.

\subsubsection{Generalization from normal distribution to EFD}

Model (14) is defined under the assumption that the conditional distributions of observed variables in $\mathbf{u}_{i}$, given latent variables, are normal. To handle non-normal data, we generalized the conditional distribution of $\mathbf{u}_{i}$ from normal to EFD. This family includes discrete distributions such as binomial and Poisson; it also includes continuous distributions such as normal and gamma.

We assume that the distribution of $u_{i j}, j=1, \ldots, p$ conditional on the $k$ th class and $\boldsymbol{\omega}_{i}$, is independent and comes from the EFD with canonical parameter $\vartheta_{k i j}[1,31,33]$ :

$$
\begin{aligned}
& p_{k j}\left(u_{i j} \mid c_{i}=k, \boldsymbol{\omega}_{i}\right) \\
& \quad=\exp \left\{\left[u_{i j} \vartheta_{k i j}-b_{j}\left(\vartheta_{k i j}\right)\right] / \psi_{\epsilon k j}+c_{j}\left(u_{i j}, \psi_{\epsilon k j}\right)\right\}
\end{aligned}
$$

where $b_{j}(\cdot)$ and $c_{j}(\cdot)$ are differentiable functions with the dots denoting the derivatives, the forms of which depend on the distributions of the response variables, $E\left(u_{i j} \mid c_{i}=\right.$ $\left.k, \boldsymbol{\omega}_{i}\right)=\dot{b}_{j}\left(\vartheta_{k i j}\right)$, and $\operatorname{Var}\left(u_{i j} \mid c_{i}=k, \boldsymbol{\omega}_{i}\right)=\psi_{\epsilon k j} \ddot{b}_{j}\left(\vartheta_{k i j}\right)$. Let $\boldsymbol{\vartheta}_{k i}=\left(\vartheta_{k i 1}, \ldots, \vartheta_{k i p}\right)^{T}$, where $\vartheta_{k i j}=g_{j}\left(E\left(u_{i j} \mid c_{i}=\right.\right.$ $\left.k, \boldsymbol{\omega}_{i}\right)$ ), and $g_{j}(\cdot)$ are link functions. Under the EFD framework, The expectation of Equation (14) can be rewritten as

$$
\boldsymbol{\vartheta}_{k i}=\boldsymbol{\Lambda}_{k} \boldsymbol{\omega}_{i}
$$

Similarly, the distribution of $\mathbf{v}_{i}$ in Equation (12) can also be generalized from normal to EFD. Let $\boldsymbol{\vartheta}_{v i}=$ $\left(\vartheta_{v i 1}, \ldots, \vartheta_{v i p_{3}}\right)^{T}$, where $\vartheta_{v i j}=h_{j}\left(E\left(v_{i j} \mid \boldsymbol{\zeta}_{i}\right)\right)$, and $h_{j}(\cdot)$ are link functions. Under the EFD framework, the expectation of Equation (12) can be rewritten as

$$
\boldsymbol{\vartheta}_{v i}=\boldsymbol{\Lambda}_{v} \boldsymbol{\zeta}_{i}
$$

For instance, in the study of the NLSY79 data, the indicators $\left\{v_{i 1}, v_{i 2}, v_{i 3}\right\}$ for measuring the latent variable "home environment, $\zeta_{i}$ " are discrete and continuous, in which $v_{i 1}$ is a binary variable coded with $\{0,1\}$ representing "in household with father and other relatives" and "in household with mother," respectively, whereas $v_{i 2}$, home cognitive stimulation score, and $v_{i 3}$, emotional support score, are continuous and assumed to be normally distributed. Under the EFD framework, the corresponding CFA model can be written as

$$
\left(\begin{array}{l}
\vartheta_{v 1} \\
\vartheta_{v 2} \\
\vartheta_{v 3}
\end{array}\right)=\left(\begin{array}{c}
1.0 \\
\lambda_{v, 21} \\
\lambda_{v, 31}
\end{array}\right) \zeta_{i},
$$

where $\vartheta_{v j}=h_{j}\left(E\left(v_{i j} \mid \zeta_{i}\right)\right)$, in which the link function $h_{1}$ is a logit link, and $h_{2}$ and $h_{3}$ are the identity links. The value of 1.0 is fixed to identify the model and to introduce a scale to the latent variable $\zeta_{i}$.

In summary, the GLCMM proposed in this section integrates the following features: (i) continuous latent variables, including latent growth factors and explanatory latent variables that may influence both latent growth curve and latent class membership; (ii) a latent allocation variable that accounts for latent trajectory classes; (iii) fixed and latent effects in LCM and in the multinomial logit model; and (iv) discrete and continuous data from EFD. Hence, the proposed model is rather general and useful for revealing possible latent classes and class-specific change patterns in the analysis of heterogeneous longitudinal data.

\section{BAYESIAN ANALYSIS OF GLCMM}

\subsection{Bayesian estimation with MCMC methods}

\subsubsection{Prior distributions}

In a full Bayesian analysis, the unknown parameters are treated as random. An important issue is to specify prior 
distributions for unknown parameters. Diebolt and Roberts [9] and Roeder and Wasserman [30] pointed out that using fully non-informative prior distributions in a mixture model may lead to improper posterior distributions. Hence, following the common practice in Bayesian mixture modeling [see, for example, 18, 30, 33], we utilize the conjugate type prior distributions in this analysis.

Let $\boldsymbol{\Lambda}_{k m}^{T}$ denote the $m$-th row of $\boldsymbol{\Lambda}_{k}, \psi_{\epsilon k m}$ denote the $m$ th diagonal element of $\boldsymbol{\Psi}_{\epsilon k}$, for $m=1,2, \ldots, p$, and $\boldsymbol{\Lambda}_{\delta k}=$ $\left(\mathbf{A}_{k}, \boldsymbol{\Pi}_{k}, \boldsymbol{\Gamma}_{k}\right)$; let $\boldsymbol{\Lambda}_{\delta k l}^{T}$ denote the $l$-th row of $\boldsymbol{\Lambda}_{\delta k}, \psi_{\delta k l}$ denote the $l$-th diagonal element of $\boldsymbol{\Psi}_{\delta k}$, for $l=1,2, \ldots, q_{1}$; $\boldsymbol{\Lambda}_{v l}^{T}$ denote the $l$-th row of $\boldsymbol{\Lambda}_{v}$, and $\psi_{v l}$ denote the $l$-th diagonal element of $\boldsymbol{\Psi}_{v}$, for $l=1,2, \ldots, p_{3}$. The following conjugate prior distributions are specified:

$$
\begin{aligned}
& p\left(\boldsymbol{\Lambda}_{k m}\right) \sim N\left(\boldsymbol{\Lambda}_{0 \epsilon k m}, \psi_{\epsilon k m} \mathbf{H}_{0 \epsilon k m}\right), \\
& p\left(\psi_{\epsilon k m}^{-1}\right) \sim \operatorname{Gamma}\left(\alpha_{0 \epsilon k m}, \beta_{0 \epsilon k m}\right), \\
& p\left(\boldsymbol{\Lambda}_{\delta k l}\right) \sim N\left(\boldsymbol{\Lambda}_{0 \delta k l}, \psi_{\delta k l} \mathbf{H}_{0 \delta k l}\right), \\
& p\left(\psi_{\delta k l}^{-1}\right) \sim \operatorname{Gamma}\left(\alpha_{0 \delta k l}, \beta_{0 \delta k l}\right), \\
& p\left(\boldsymbol{\Lambda}_{v l}\right) \sim N\left(\boldsymbol{\Lambda}_{0 v l}, \psi_{v l} \mathbf{H}_{0 v l}\right), \\
& p\left(\psi_{v l}^{-1}\right) \sim \operatorname{Gamma}\left(\alpha_{0 v l}, \beta_{0 v l}\right), \\
& p\left(\boldsymbol{\mu}_{k}\right) \sim N\left(\boldsymbol{\mu}_{0 k}, \boldsymbol{\Sigma}_{0 k}\right), \\
& p\left(\boldsymbol{\Phi}_{k}^{-1}\right) \sim \text { Wishart }\left(\mathbf{R}_{0 k}, \rho_{0}\right), \\
& p\left(\boldsymbol{\varphi}_{k}\right) \sim N\left(\boldsymbol{\varphi}_{0 k}, \boldsymbol{\Sigma}_{\varphi 0 k}\right), \\
& p\left(\boldsymbol{\Phi}_{v}^{-1}\right) \sim \text { Wishart }\left(\mathbf{R}_{0 v}, \rho_{0 v}\right),
\end{aligned}
$$

where $\boldsymbol{\Lambda}_{0 \epsilon k m}, \boldsymbol{\Lambda}_{0 \delta k l}, \boldsymbol{\Lambda}_{0 v l}, \boldsymbol{\mu}_{0 k}, \boldsymbol{\varphi}_{0 k}, \alpha_{0 \epsilon k m}, \beta_{0 \epsilon k m}, \alpha_{0 \delta k l}$, $\beta_{0 \delta k l}, \alpha_{0 v l}, \beta_{0 v l}, \rho_{0}$ and $\rho_{0 v}$, and the positive-definite matrices $\mathbf{H}_{0 \epsilon k m}, \mathbf{H}_{0 \delta k l}, \mathbf{H}_{0 v l}, \boldsymbol{\Sigma}_{0 k}, \boldsymbol{\Sigma}_{\varphi 0 k}, \mathbf{R}_{0 k}$, and $\mathbf{R}_{0 v}$ are hyperparameters. In practice, values of hyperparameters are adopted from previous studies or other sources. Given that assigning conjugate prior distributions for unknown parameters usually leads to the same forms of posterior distributions $[11,13,18]$, the resulting posterior distributions for most of the unknown parameters in our proposed model are standard, and simulating observations from them is efficient.

\subsubsection{Posterior inferences with MCMC sampling}

Let $\mathbf{U}=\left(\mathbf{u}_{1}, \mathbf{u}_{2}, \ldots, \mathbf{u}_{n}\right)$ and $\mathbf{V}=\left(\mathbf{v}_{1}, \mathbf{v}_{2}, \ldots, \mathbf{v}_{n}\right)$ denote the observed data matrices, $\boldsymbol{\Omega}=\left(\boldsymbol{\omega}_{1}, \boldsymbol{\omega}_{2}, \ldots, \boldsymbol{\omega}_{n}\right)$ and $\widetilde{\boldsymbol{\Omega}}=\left(\boldsymbol{\zeta}_{1}, \boldsymbol{\zeta}_{2}, \ldots, \boldsymbol{\zeta}_{n}\right)$ denote the matrices of continuous latent variables, and $\mathbf{C}=\left(c_{1}, c_{2}, \ldots, c_{n}\right)^{T}$ denote the vector of latent allocation variables. Moreover, let $\boldsymbol{\theta}_{k}$ denote the parameter vector that contains all the unknown parameters in $\boldsymbol{\Lambda}_{k}, \boldsymbol{\mu}_{k}, \mathbf{A}_{k}, \boldsymbol{\Pi}_{k}, \boldsymbol{\Gamma}_{k}, \boldsymbol{\Phi}_{k}, \boldsymbol{\Psi}_{\epsilon k}, \boldsymbol{\Psi}_{\delta k}, \boldsymbol{\Lambda}_{v}, \boldsymbol{\Phi}_{v}, \boldsymbol{\Psi}_{v}$, and $\boldsymbol{\varphi}_{k}$, where $\boldsymbol{\varphi}_{k}=\left(\varphi_{k 0}, \varphi_{k 1}, \ldots, \varphi_{k, r_{2}+q_{3}}\right)^{T}$, for $k=1,2, \ldots, K$. Denote $\boldsymbol{\theta}=\left\{\boldsymbol{\theta}_{1}, \boldsymbol{\theta}_{2}, \ldots, \boldsymbol{\theta}_{K}\right\}$. Considering that the posterior distribution of $\boldsymbol{\theta}$ given $\mathbf{U}$ and $\mathbf{V}, p(\boldsymbol{\theta} \mid \mathbf{U}, \mathbf{V})$, is intractable, we performed data augmentation [40] by augmenting the observed data $\mathbf{F}_{o}=\{\mathbf{U}, \mathbf{V}\}$ with the latent quantities $\mathbf{F}_{l}=\{\boldsymbol{\Omega}, \widetilde{\boldsymbol{\Omega}}, \mathbf{C}\}$ in the posterior analysis. The Bayesian estimate of $\boldsymbol{\theta}$ is obtained based on the observations drawn from $p\left(\boldsymbol{\theta}, \mathbf{F}_{l} \mid \mathbf{F}_{o}\right)$ using MCMC tools such as the Gibbs sampler and the MH algorithm. The Bayesian analysis of the proposed model is complex. In particular, the following issues should be addressed.

(1) Posterior distributions and sampling Given the complexity of the model and data structure, some full conditional distributions involved in the MCMC algorithm are nontrivial. For instance, the EFD framework, the nonlinear function $\mathbf{F}(\cdot)$, and the multinomial logit model lead to the posterior distributions that one cannot directly sample from (see details in the Appendix). Simulating observations from these distributions is a challenging task in implementing the MCMC algorithm. We employed the MH algorithm together with other MCMC techniques to achieve this purpose. The full conditional distributions of other parameters in $\boldsymbol{\theta}$ are the normal, Gamma, and inverted Wishart distributions. Simulating observations from these distributions is straightforward.

(2) Label switching and permutation sampler A second issue that needs to be addressed is the label switching problem in Bayesian mixture modeling. For the proposed GLCMM with $K$ classes, the likelihood function is invariant with a permutation of the class labels $1,2, \ldots, K$. So, the unconstrained posterior is also invariant to relabeling the states under a symmetric prior. This condition induces a multimodal posterior, and may give rise to misleading results in Bayesian estimation. Following Lee [18], the permutation sampler proposed by Frühwirth-Schnatter [14] is used to solve the label switching problem. The details of permutation sampler can be found in [14] and [18].

To conduct the posterior inference, a sufficiently large number of random observations were simulated from the joint posterior distribution $p\left(\boldsymbol{\theta}, \mathbf{F}_{l} \mid \mathbf{F}_{o}\right)$ using the Gibbs sampler [15] coupled with the MH algorithm [17, 25]. More specifically, $\boldsymbol{\theta}$ and $\mathbf{F}_{l}$ were simulated from their corresponding full conditional distributions iteratively. Under mild regularity conditions, the joint distribution of $\left(\boldsymbol{\theta}^{(j)}, \mathbf{F}_{l}^{(j)}\right)$ converges at an exponential rate to the desired posterior distribution $p\left(\boldsymbol{\theta}, \mathbf{F}_{l} \mid \mathbf{F}_{o}\right)$, after a sufficiently large number of burnin iterations [15]. The required number of burn-in iterations for achieving convergence can be determined by plotting the simulated sequences of individual parameters. At convergence, parallel sequences generated with different starting values should mix well. Another method for monitoring convergence is based on the estimated potential scale reduction (EPSR) values [16], in which convergence is achieved when the EPSR values are all less than 1.2. Based on a simulated sample of observations after convergence, the Bayesian estimates of $\boldsymbol{\theta}$ can be obtained through the sample mean and the standard error estimates can be obtained through the sample covariance matrix.

\subsection{Bayesian model selection}

For the proposed GLCMM, the number of latent classes, $K$, is usually unknown. A model selection procedure is 
needed to determine the most appropriate $K$. In this article, we adopted a modified DIC [38] for the model selection. DIC combines Bayesian measures of fit and model complexity. The model with the smallest DIC value is selected. However, DIC cannot be directly applied to the selection of mixture models [18, 39]. Recently, Celeux et al. [6] have proposed different DIC constructions for mixture models. In this paper, the most current modification of DIC for mixture model is used. This modified DIC is defined as follows:

$$
\begin{aligned}
D I C= & -4 E_{\boldsymbol{\theta}, \mathbf{F}_{l}}\left\{\log p\left(\mathbf{F}_{o}, \mathbf{F}_{l} \mid \boldsymbol{\theta}\right) \mid \mathbf{F}_{o}\right\} \\
& +2 E_{\mathbf{F}_{l}}\left\{\log p\left(\mathbf{F}_{o}, \mathbf{F}_{l} \mid E_{\boldsymbol{\theta}}\left[\boldsymbol{\theta} \mid \mathbf{F}_{o}, \mathbf{F}_{l}\right]\right) \mid \mathbf{F}_{o}\right\}
\end{aligned}
$$

where $\log p\left(\mathbf{F}_{o}, \mathbf{F}_{l} \mid \boldsymbol{\theta}\right)$ is the complete data log-likelihood function. The modified DIC can be approximated by

$D I C$

$$
\approx-\frac{4}{T} \sum_{t=1}^{T} \log p\left(\mathbf{F}_{o}, \mathbf{F}_{l}^{(t)} \mid \boldsymbol{\theta}^{(t)}\right)+\frac{2}{T} \sum_{t=1}^{T} \log p\left(\mathbf{F}_{o}, \mathbf{F}_{l}^{(t)} \mid \overline{\boldsymbol{\theta}}^{(t)}\right)
$$

where $\left\{\left(\boldsymbol{\theta}^{(t)}, \mathbf{F}_{l}^{(t)}\right) ; t=1, \ldots, T\right\}$ are the simulated samples from $p\left(\boldsymbol{\theta}, \mathbf{F}_{l} \mid \cdot\right)$, and $\overline{\boldsymbol{\theta}}^{(t)}$ is the sample mean calculated based on the simulated samples from $p\left(\boldsymbol{\theta} \mid \mathbf{F}_{o}, \mathbf{F}_{l}^{(t)}\right)$.

\section{SIMULATION STUDY}

The objective of this simulation study is to examine the empirical performance of the Bayesian estimation and model selection for the proposed GLCMM. The data set was generated from a GLCMM with two trajectory classes $(K=2)$. For $k=1,2$, and $i=1,2, \ldots, n$, the probability of the $i$ th individual falling in the $k$ th class was modeled through the following multinomial logit model with $r_{2}=1, q_{3}=2$ :

$$
\pi_{i k}=\frac{\exp \left(\varphi_{k 0}+\varphi_{k 1} b_{i}+\varphi_{k 2} \zeta_{i 1}+\varphi_{k 3} \zeta_{i 2}\right)}{\sum_{l=1}^{2} \exp \left(\varphi_{l 0}+\varphi_{l 1} b_{i}+\varphi_{l 2} \zeta_{i 1}+\varphi_{l 3} \zeta_{i 2}\right)},
$$

where $\left(\varphi_{10}, \varphi_{11}, \varphi_{12}, \varphi_{13}\right)^{T}=(-0.5,1.0,0.8,0.8)^{T}$, and $\left(\varphi_{20}, \varphi_{21}, \varphi_{22}, \varphi_{23}\right)^{T}$ is fixed to $(0.0,0.0,0.0,0.0)^{T}$ for identifying the model; the fixed covariate $b_{i}$ was generated from $N(1.0,1.0)$; and the explanatory latent variables $\left(\zeta_{i 1}, \zeta_{i 2}\right)$ were measured by six observed indicators via the following CFA model:

$$
\left(\begin{array}{l}
v_{i 1} \\
v_{i 2} \\
v_{i 3} \\
v_{i 4} \\
v_{i 5} \\
v_{i 6}
\end{array}\right)=\left(\begin{array}{cc}
1.0 & 0.0 \\
\lambda_{v, 21} & 0.0 \\
\lambda_{v, 31} & 0.0 \\
0.0 & 1.0 \\
0.0 & \lambda_{v, 52} \\
0.0 & \lambda_{v, 62}
\end{array}\right)\left(\begin{array}{l}
\zeta_{i 1} \\
\zeta_{i 2}
\end{array}\right)+\left(\begin{array}{c}
\epsilon_{v i 1} \\
\epsilon_{v i 2} \\
\epsilon_{v i 3} \\
\epsilon_{v i 4} \\
\epsilon_{v i 5} \\
\epsilon_{v i 6}
\end{array}\right)
$$

where 1's and 0's are fixed to identify the model, $\left(\zeta_{i 1}, \zeta_{i 2}\right)^{T} \sim N\left(\mathbf{0}, \Phi_{v}\right)$, and $\epsilon_{v i j} \sim N\left(0, \psi_{v j}\right)$. The true population values of unknown parameters are $\lambda_{v, 21}=\lambda_{v, 31}=$ $\lambda_{v, 52}=\lambda_{v, 62}=0.8, \phi_{v, 11}=\phi_{v, 22}=1.0$, and $\phi_{v, 21}=0.3$, and $\psi_{v 1}=\cdots=\psi_{v 6}=0.36$.

To define the within-class generalized LCM, we let $\mathbf{u}_{i}=$ $\left(u_{i 1}, u_{i 2}, \ldots, u_{i, 14}\right)^{T}=\left(y_{i 1}, \ldots, y_{i 8}, x_{i 1}, \ldots, x_{i 6}\right)^{T}$. For $j=$ $1, \ldots, 4, y_{i j}$ were generated from a binomial distribution $B\left(1, p_{k i j}\right)$, with $p_{k i j}=\exp \left(\vartheta_{k i j}\right) /\left[1+\exp \left(\vartheta_{k i j}\right)\right]$, and $\vartheta_{k i j}$ satisfy the LCM

$$
\left(\begin{array}{l}
\vartheta_{k i 1} \\
\vartheta_{k i 1} \\
\vartheta_{k i 3} \\
\vartheta_{k i 4}
\end{array}\right)=\left(\begin{array}{ll}
1.0 & 0.0 \\
1.0 & 1.0 \\
1.0 & 2.0 \\
1.0 & 3.0
\end{array}\right)\left(\begin{array}{l}
\eta_{i 1} \\
\eta_{i 2}
\end{array}\right)
$$

For $j=5, \ldots, 8, y_{i j}$ are continuous, and satisfy the LCM

$$
\left(\begin{array}{l}
y_{i 5} \\
y_{i 6} \\
y_{i 7} \\
y_{i 8}
\end{array}\right)=\left(\begin{array}{ll}
1.0 & 0.0 \\
1.0 & 1.0 \\
1.0 & 2.0 \\
1.0 & 3.0
\end{array}\right)\left(\begin{array}{l}
\eta_{i 3} \\
\eta_{i 4}
\end{array}\right)+\left(\begin{array}{l}
\epsilon_{y i 5} \\
\epsilon_{y i 6} \\
\epsilon_{v i 7} \\
\epsilon_{v i 8}
\end{array}\right)
$$

where $\epsilon_{y i j} \sim N\left(0, \psi_{y k j}\right)$.

For $j=1, \ldots, 6, x_{i j}$ relate to the explanatory latent variables $\left(\xi_{i 1}, \xi_{i 2}\right)$ via the CFA model

$$
\left(\begin{array}{l}
x_{i 1} \\
x_{i 2} \\
x_{i 3} \\
x_{i 4} \\
x_{i 5} \\
x_{i 6}
\end{array}\right)=\left(\begin{array}{cc}
1.0 & 0.0 \\
\lambda_{x k, 21} & 0.0 \\
\lambda_{x k, 31} & 0.0 \\
0.0 & 1.0 \\
0.0 & \lambda_{x k, 52} \\
0.0 & \lambda_{x k, 62}
\end{array}\right)\left(\begin{array}{l}
\xi_{i 1} \\
\xi_{i 2}
\end{array}\right)+\left(\begin{array}{c}
\epsilon_{x i 1} \\
\epsilon_{x i 2} \\
\epsilon_{x i 3} \\
\epsilon_{x i 4} \\
\epsilon_{x i 5} \\
\epsilon_{x i 6}
\end{array}\right)
$$

where 1's and 0's are fixed for the purpose of identification, $\left(\xi_{i 1}, \xi_{i 2}\right)^{T} \sim N\left(\mathbf{0}, \boldsymbol{\Phi}_{k}\right)$, and $\epsilon_{x i j} \sim N\left(0, \psi_{x k j}\right)$.

The effects of explanatory latent variables and covariates on the latent growth factors, $\eta_{i j}, j=1, \ldots, 4$ were assessed through the structural equations with $r_{1}=1, q_{2}=2$, and $\mathbf{F}\left(\boldsymbol{\xi}_{i}\right)=\left(\xi_{i 1}, \xi_{i 2}, \xi_{i 1} \xi_{i 2}\right)^{T}$ as follows:

$$
\begin{aligned}
& \eta_{i 1}=\mu_{k 1}+a_{k, 11} w_{i}+\gamma_{k, 11} \xi_{i 1}+\gamma_{k, 12} \xi_{i 2}+\gamma_{k, 13} \xi_{i 1} \xi_{i 2}+\delta_{i 1} \\
& \eta_{i 2}=\mu_{k 2}+a_{k, 21} w_{i}+\gamma_{k, 21} \xi_{i 1}+\gamma_{k, 22} \xi_{i 2}+\gamma_{k, 23} \xi_{i 1} \xi_{i 2}+\delta_{i 2} \\
& \eta_{i 3}=\mu_{k 3}+a_{k, 31} w_{i}+\gamma_{k, 31} \xi_{i 1}+\gamma_{k, 32} \xi_{i 2}+\gamma_{k, 33} \xi_{i 1} \xi_{i 2}+\delta_{i 3} \\
& \eta_{i 4}=\mu_{k 4}+a_{k, 41} w_{i}+\gamma_{k, 41} \xi_{i 1}+\gamma_{k, 42} \xi_{i 2}+\gamma_{k, 43} \xi_{i 1} \xi_{i 2}+\delta_{i 4}
\end{aligned}
$$

where the $\mu$ 's, $a$ 's, and $\gamma$ 's were unknown parameters. The fixed covariate $w_{i}(i=1,2, \ldots, n)$ was generated from $N(0.0,1.0)$, and $\delta_{i j} \sim N\left(0, \psi_{\delta k j}\right)$.

Figure 4 depicts the path diagram of the within-class generalized LCM defined above.

In Figure 4, two linear LCMs were considered simultaneously. One was defined with latent growth factors $\left\{\eta_{i 1}, \eta_{i 2}\right\}$ and binary repeated measures $y_{i 1}, \ldots, y_{i 4}$ under the EFD framework (see Equation (17)). The other was defined with latent growth factors $\left\{\eta_{i 3}, \eta_{i 4}\right\}$ and continuous repeated measures $y_{i 5}, \ldots, y_{i 8}$.

The true population values of the unknown parameters involved in the within-class LCMs are given as follows: 


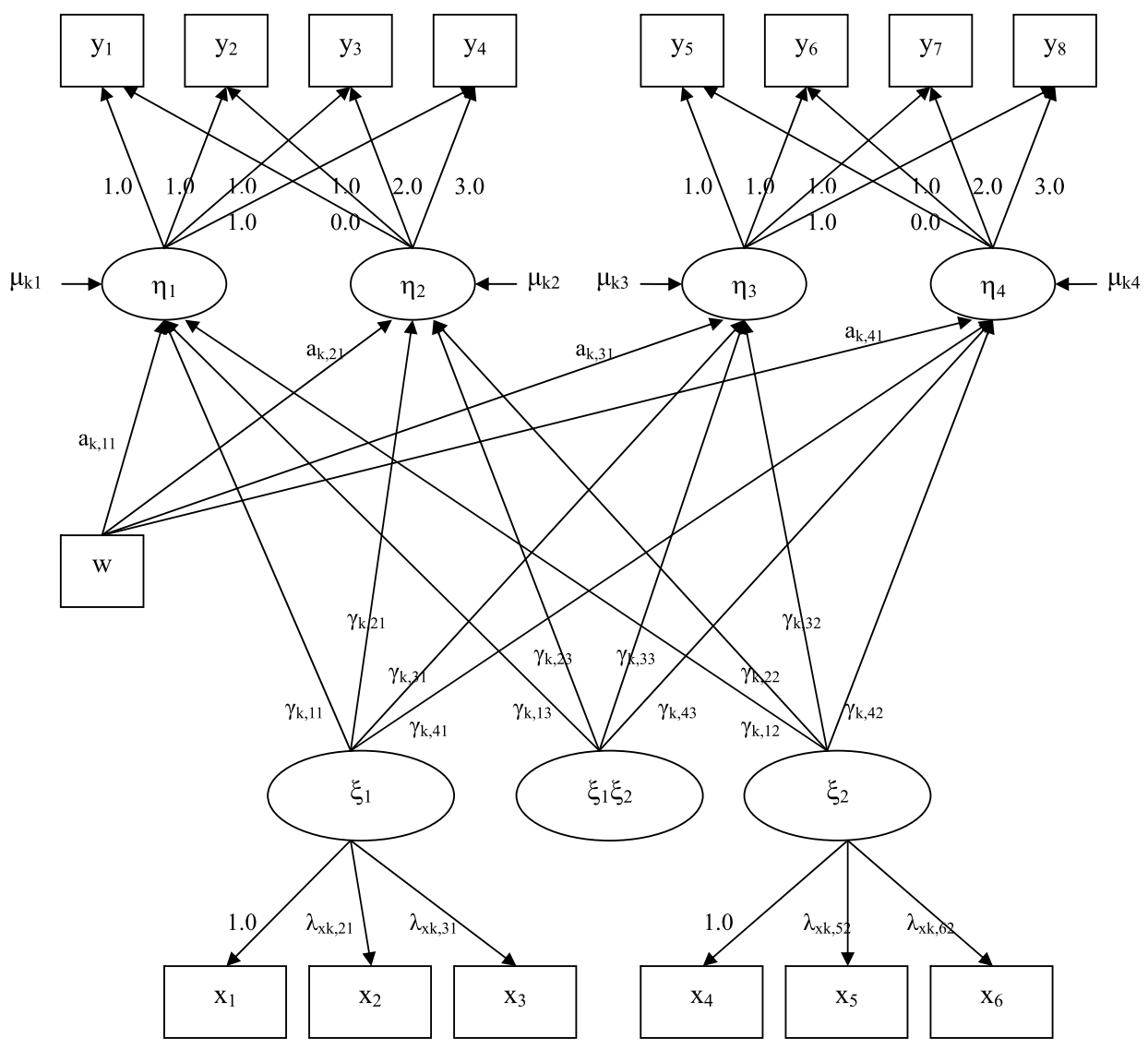

Figure 4. Path diagram of within-class generalized LCM for simulation study.

At the first class $(k=1), \lambda_{x 1,21}=\lambda_{x 1,31}=\lambda_{x 1,52}=$ $\lambda_{x 1,62}=0.8 ;\left(\mu_{11}, \mu_{12}, \mu_{13}, \mu_{14}\right)^{T}=(2.0,1.0,-2.0,-1.5)^{T} ;$ $\left(a_{1,11}, a_{1,21}, a_{1,31}, a_{1,41}\right)^{T}=(0.5,-0.5,0.5,-0.5)^{T} ; \gamma_{1, j m}=$ 0.5 , for $j=1, \ldots, 4, m=1, \ldots, 3 ; \psi_{y 1 j}=0.36$, for $j=$ $5, \ldots, 8 ; \psi_{x 1 j}=0.36$, for $j=1, \ldots, 6 ; \psi_{\delta 1 l}=0.36$, for $l=$ $1, \ldots, 4 ; \phi_{1,11}=\phi_{1,22}=1.0$, and $\phi_{1,21}=0.3$.

At the second class $(k=2), \lambda_{x 2,21}=\lambda_{x 2,31}=\lambda_{x 2,52}=$ $\lambda_{x 2,62}=0.4 ;\left(\mu_{21}, \mu_{22}, \mu_{23}, \mu_{24}\right)^{T}=(1.0,1.5,1.5,0.5)^{T}$; $\left(a_{2,11}, a_{2,21}, a_{1,31}, a_{2,41}\right)^{T}=(-0.5,0.5,-0.5,0.5)^{T} ; \gamma_{2, j m}=$ -0.5 , for $j=1, \ldots, 4, m=1, \ldots, 3 ; \psi_{y 2 j}=0.25$, for $j=5, \ldots, 8 ; \psi_{x 2 j}=0.25$, for $j=1, \ldots, 6 ; \psi_{\delta 2 l}=0.25$, for $l=1, \ldots, 4 ; \phi_{2,11}=\phi_{2,22}=1.0$, and $\phi_{2,21}=0.3$.

The total number of unknown parameters in the proposed model was 99. Given the number of parameters and the existence of binary data, a relatively large sample size was required to achieve accurate estimation result [18]. In this simulation study, sample sizes of $n=800$ and $n=1,600$ were used, and 100 replications were conducted for each sample size.

To provide a sensitivity analysis of the Bayesian results to the prior specification, the prior input in (20) was perturbed as follows:

Type I: All elements in $\left\{\boldsymbol{\mu}_{0 k}, \boldsymbol{\Lambda}_{0 \epsilon k m}, \boldsymbol{\Lambda}_{0 \delta k l}, \boldsymbol{\varphi}_{0 k}, \boldsymbol{\Lambda}_{0 v l}\right\}$ were taken to be 1.0, $\alpha_{0 \epsilon k m}=\alpha_{0 \delta k l}=9, \beta_{0 \epsilon k m}=\beta_{0 \delta k l}=4$, $\boldsymbol{\Sigma}_{0 k}, \mathbf{H}_{0 \epsilon k m}, \mathbf{H}_{0 \delta k l}, \mathbf{H}_{0 v l}$ and $\boldsymbol{\Sigma}_{\varphi 0 k}$ were identity matrices,

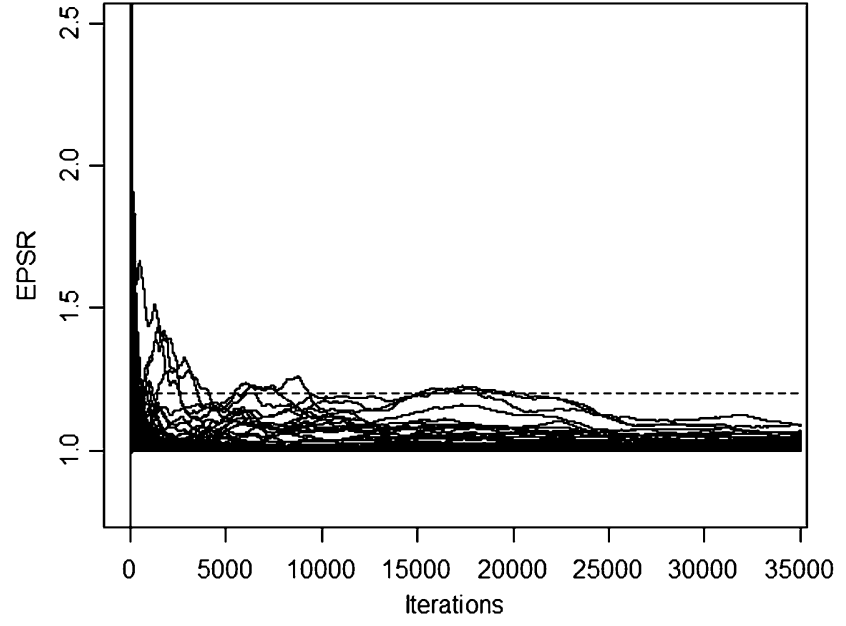

Figure 5. Plot of the estimated potential scale reduction values against the iteration numbers in simulation study.

$\rho_{0}=\rho_{0 v}=5, \mathbf{R}_{0 k}^{-1}$ and $\mathbf{R}_{0 v}^{-1}$ were taken as diagonal matrix with diagonal elements 2.0.

Type II: All elements in $\left\{\boldsymbol{\mu}_{0 k}, \boldsymbol{\Lambda}_{0 \epsilon k m}, \boldsymbol{\Lambda}_{0 \delta k l}, \boldsymbol{\varphi}_{0 k}, \boldsymbol{\Lambda}_{0 v l}\right\}$ were taken to be 0.0, $\alpha_{0 \epsilon k m}=\alpha_{0 \delta k l}=10, \beta_{0 \epsilon k m}=\beta_{0 \delta k l}=$ $3, \boldsymbol{\Sigma}_{0 k}, \mathbf{H}_{0 \epsilon k m}, \mathbf{H}_{0 \delta k l}, \mathbf{H}_{0 v l}$ and $\boldsymbol{\Sigma}_{\varphi 0 k}$ were diagonal matrices 

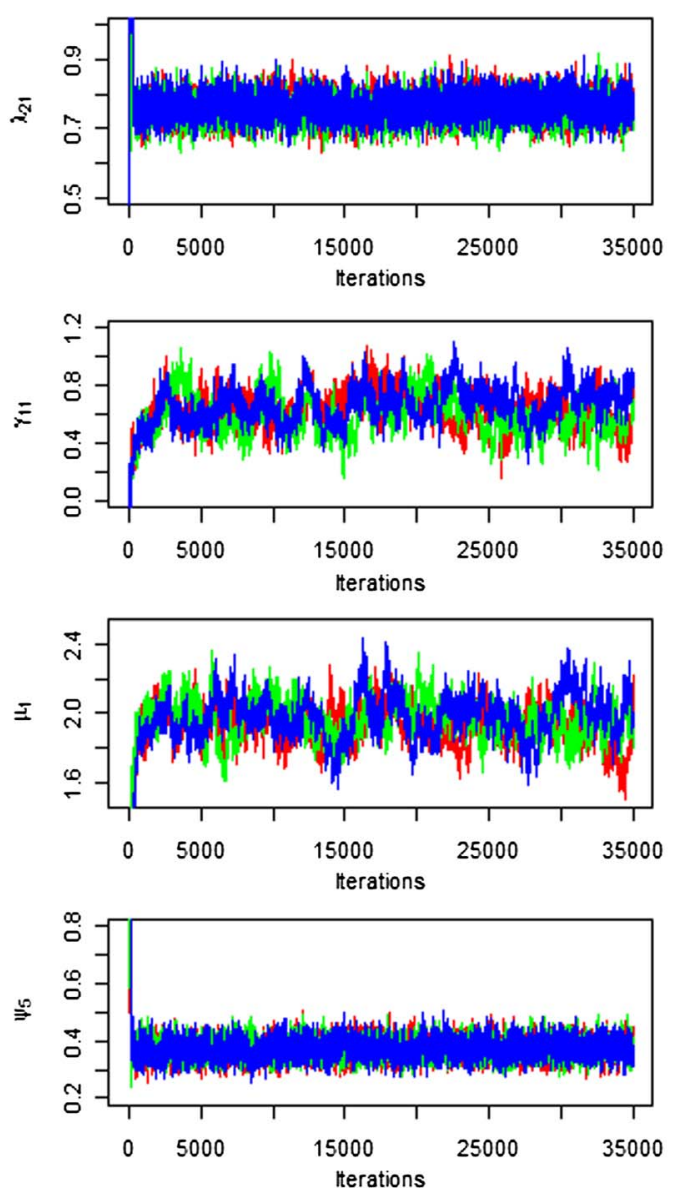
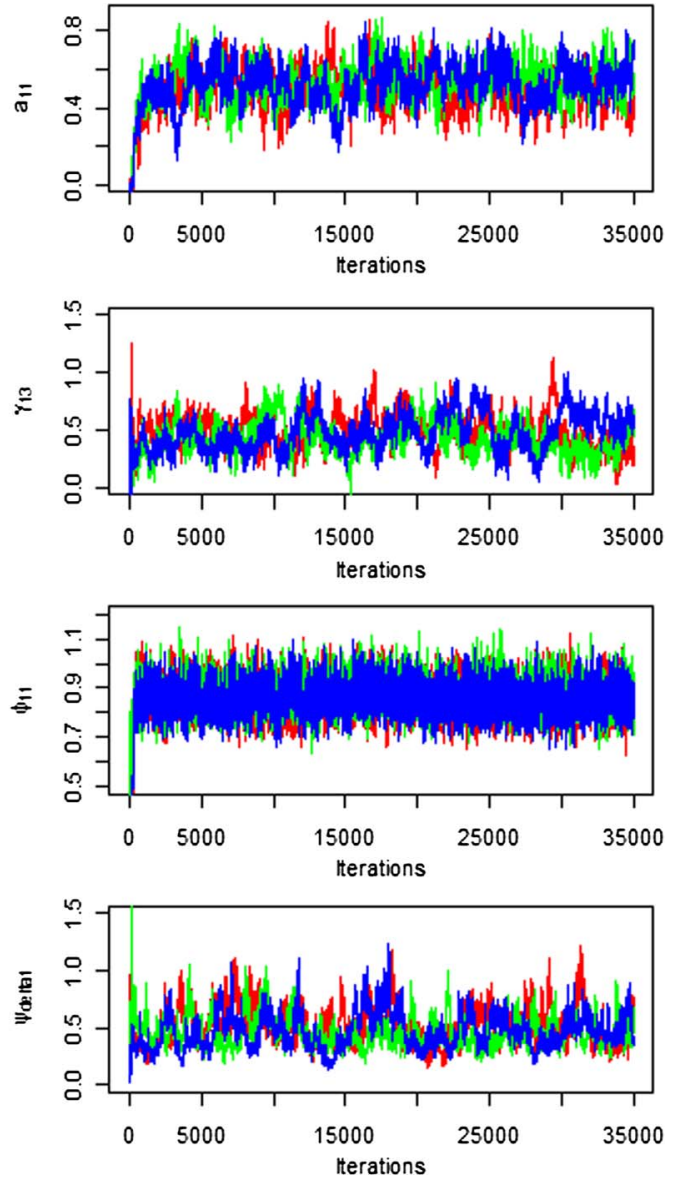

Figure 6. Three chains of observations corresponding to $\lambda_{21}, a_{11}, \gamma_{11}, \gamma_{13}, \mu_{1}, \phi_{11}, \psi_{5}$ and $\psi_{\delta 1}$ of Class 1 in simulation study generated by different initial values.

with diagonal elements $5.0, \rho_{0}=\rho_{0 v}=8, \mathbf{R}_{0 k}^{-1}$ and $\mathbf{R}_{0 v}^{-1}$ were taken as diagonal matrices with diagonal elements 8.0.

Initially, we ran the random permutation sampler [14] and found that $\mu_{13}<\mu_{23}$ was an appropriate identifiability constraint for the MCMC algorithm. A few test runs were conducted to provide information about the number of MCMC iterations required for achieving convergence. For $n=800$, Figure 5 shows that the MCMC algorithm converged in less than 25,000 iterations, in which the EPSR values were stable below 1.2. Moreover, the plots of sequences of observations corresponding to the parameters randomly selected from Class 1 are displayed in Figure 6. Those corresponding to other parameters are very similar and are not presented here. These trace plots show that MCMC chains with different starting values mix very well. Hence, we collected 10,000 observations after discarding 25,000 burn-in iterations to obtain the Bayesian results at each replication. Based on 100 replications, the bias of the estimates (Bias) and the root mean squares (RMS) between the estimates and the true values were computed. Tables 1 to 4 report the results. The findings are summarized as follows: (1) Most of the Bias and the RMS values are reasonably small. (2) The estimates of the parameters associated with the binary observed variables are not as accurate as those associated with the continuous observed variables (i.e., their RMS values are relatively larger) because binary variables usually provide less information than continuous variables. (3) Under the given sample sizes, the Bayesian estimates obtained under the two different prior inputs have no substantial difference. (4) As expected, a larger sample size produces better estimates with smaller RMS values.

To access the performance of the modified DIC in determining the number of classes, $K$, we denote $M_{k}$ as the GLCMM with $k$ latent classes, $k=1,2,3$. Here, $M_{2}$ is the true model defined above. For $M_{1}$ and $M_{3}$, the model setting and prior specification in each class are similar to those given in $M_{2}$. The modified DIC values for $M_{1}$ to $M_{3}$ were calculated from the simulated observations in the MCMC iterations. Table 5 presents a summary of the modified DIC values under different model settings and prior inputs. With $n=800$, the true model $M_{2}$ was selected with the smallest DIC value in 98 of 100 replications under Prior I and 96 of 100 replications under Prior II. With $n=1,600$, the true model $M_{2}$ is selected with the smallest DIC value under each 
Table 1. Performance of the Bayesian estimates in the simulation study with Type I prior input under sample size $n=800$

\begin{tabular}{|c|c|c|c|c|c|c|c|c|c|c|c|c|c|c|}
\hline & & Class 1 & & & Class & & & & & Class 1 & & & Class 2 & \\
\hline Par & True & Bias & RMS & True & Bias & RMS & & Par & True & Bias & RMS & True & Bias & RMS \\
\hline$\overline{\lambda_{21}}$ & 0.8 & 0.012 & 0.044 & 0.4 & 0.024 & 0.044 & & $\psi_{y 5}$ & 0.36 & 0.008 & 0.039 & 0.25 & 0.023 & 0.035 \\
\hline$\lambda_{31}$ & 0.8 & 0.019 & 0.049 & 0.4 & 0.024 & 0.044 & & $\psi_{y 6}$ & 0.36 & 0.007 & 0.032 & 0.25 & 0.015 & 0.028 \\
\hline$\lambda_{52}$ & 0.8 & 0.013 & 0.049 & 0.4 & 0.015 & 0.048 & & $\psi_{y 7}$ & 0.36 & 0.001 & 0.034 & 0.25 & 0.009 & 0.028 \\
\hline$\lambda_{62}$ & 0.8 & 0.012 & 0.045 & 0.4 & 0.020 & 0.045 & & $\psi_{y 8}$ & 0.36 & 0.025 & 0.061 & 0.25 & 0.046 & 0.058 \\
\hline$\mu_{1}$ & 2.0 & -0.005 & 0.145 & 1.0 & 0.068 & 0.164 & & $\psi_{x 1}$ & 0.36 & 0.059 & 0.093 & 0.25 & 0.063 & 0.078 \\
\hline$\mu_{2}$ & 1.0 & 0.033 & 0.175 & 1.5 & 0.233 & 0.305 & & $\psi_{x 2}$ & 0.36 & 0.007 & 0.030 & 0.25 & 0.008 & 0.022 \\
\hline$\mu_{3}$ & -2.0 & -0.027 & 0.059 & 1.5 & 0.018 & 0.050 & & $\psi_{x 3}$ & 0.36 & 0.009 & 0.032 & 0.25 & 0.010 & 0.025 \\
\hline$\mu_{4}$ & -1.5 & -0.043 & 0.062 & 0.5 & 0.015 & 0.045 & & $\psi_{x 4}$ & 0.36 & 0.055 & 0.086 & 0.25 & 0.049 & 0.067 \\
\hline$a_{11}$ & 0.5 & -0.018 & 0.136 & -0.5 & -0.041 & 0.140 & & $\psi_{x 5}$ & 0.36 & 0.012 & 0.031 & 0.25 & 0.012 & 0.023 \\
\hline$a_{21}$ & -0.5 & -0.014 & 0.107 & 0.5 & 0.059 & 0.142 & & $\psi_{x 6}$ & 0.36 & 0.006 & 0.035 & 0.25 & 0.008 & 0.021 \\
\hline$a_{31}$ & 0.5 & -0.004 & 0.043 & -0.5 & 0.001 & 0.048 & & $\psi_{\delta 1}$ & 0.36 & 0.154 & 0.207 & 0.25 & 0.170 & 0.178 \\
\hline$a_{41}$ & -0.5 & 0.002 & 0.031 & 0.5 & -0.004 & 0.035 & & $\psi_{\delta 2}$ & 0.36 & 0.129 & 0.163 & 0.25 & 0.145 & 0.160 \\
\hline$\gamma_{11}$ & 0.5 & 0.007 & 0.161 & -0.5 & -0.042 & 0.192 & & $\psi_{\delta 3}$ & 0.36 & 0.015 & 0.050 & 0.25 & 0.019 & 0.039 \\
\hline$\gamma_{12}$ & 0.5 & -0.002 & 0.193 & -0.5 & -0.066 & 0.182 & & $\psi_{\delta 4}$ & 0.36 & 0.026 & 0.048 & 0.25 & 0.009 & 0.028 \\
\hline$\gamma_{13}$ & 0.5 & 0.147 & 0.257 & -0.5 & -0.062 & 0.200 & & $\phi_{11}$ & 1.0 & -0.038 & 0.113 & 1.0 & -0.084 & 0.129 \\
\hline$\gamma_{21}$ & 0.5 & 0.013 & 0.182 & -0.5 & -0.092 & 0.223 & & $\phi_{21}$ & 0.3 & -0.009 & 0.051 & 0.3 & -0.039 & 0.073 \\
\hline$\gamma_{22}$ & 0.5 & 0.011 & 0.154 & -0.5 & -0.099 & 0.208 & & $\phi_{22}$ & 1.0 & -0.019 & 0.094 & 1.0 & -0.076 & 0.134 \\
\hline$\gamma_{23}$ & 0.5 & 0.101 & 0.233 & -0.5 & -0.057 & 0.192 & & $\varphi_{0}$ & -0.5 & 0.016 & 0.120 & $0.0^{*}$ & & \\
\hline$\gamma_{31}$ & 0.5 & 0.015 & 0.063 & -0.5 & -0.011 & 0.059 & & $\varphi_{1}$ & 1.0 & -0.007 & 0.102 & $0.0^{*}$ & & \\
\hline$\gamma_{32}$ & 0.5 & -0.007 & 0.058 & -0.5 & 0.008 & 0.055 & & $\varphi_{2}$ & 0.8 & 0.032 & 0.124 & $0.0^{*}$ & & \\
\hline$\gamma_{33}$ & 0.5 & 0.047 & 0.083 & -0.5 & -0.057 & 0.120 & & $\varphi_{3}$ & 0.8 & 0.001 & 0.115 & $0.0^{*}$ & & \\
\hline$\gamma_{41}$ & 0.5 & 0.004 & 0.060 & -0.5 & 0.000 & 0.050 & & & & & & & & \\
\hline$\gamma_{42}$ & 0.5 & 0.001 & 0.065 & -0.5 & -0.004 & 0.050 & & & & & & & & \\
\hline$\gamma_{43}$ & 0.5 & 0.068 & 0.095 & -0.5 & -0.047 & 0.099 & & & & & & & & \\
\hline$\overline{\mathrm{Par}}$ & True & Bias & RMS & Par & True & Bias & RMS & & & & & & & \\
\hline$\overline{\lambda_{v, 21}}$ & 0.8 & 0.005 & 0.034 & $\psi_{v 1}$ & 0.36 & 0.009 & 0.037 & & & & & & & \\
\hline$\lambda_{v, 31}$ & 0.8 & 0.004 & 0.036 & $\psi_{v 2}$ & 0.36 & 0.002 & 0.025 & & & & & & & \\
\hline$\lambda_{v, 52}$ & 0.8 & 0.012 & 0.035 & $\psi_{v 3}$ & 0.36 & 0.001 & 0.025 & & & & & & & \\
\hline$\lambda_{v, 62}$ & 0.8 & 0.003 & 0.035 & $\psi_{v 4}$ & 0.36 & 0.011 & 0.033 & & & & & & & \\
\hline$\phi_{v, 11}$ & 1.0 & 0.000 & 0.078 & $\psi_{v 5}$ & 0.36 & -0.001 & 0.020 & & & & & & & \\
\hline$\phi_{v, 21}$ & 0.3 & -0.003 & 0.041 & $\psi_{v 6}$ & 0.36 & 0.003 & 0.024 & & & & & & & \\
\hline$\phi_{v, 22}$ & 1.0 & -0.001 & 0.075 & & & & & & & & & & & \\
\hline
\end{tabular}

Note: The subscript $k$ is suppressed for notational simplicity, and the 0's with asterisk are fixed for identification purpose.

of the 100 replications and each of the prior inputs. The above findings demonstrates that DIC is a valid Bayesian model selection criterion.

\section{AN ILLUSTRATIVE EXAMPLE}

In this section, we applied the GLCMM to a data set extracted from the NLSY79 data to illustrate our methodology. A subset of the NLSY79 data collected in 1990, 1992, and 1994 was used in this example. After excluding missing data, the sample size was $n=1,674$. We consider the continuous longitudinal variables $y_{1}, y_{2}, y_{3}$, which represent the PIAT scores [10] in mathematics in 1990, 1992, and 1994, respectively. PIAT is one of the most commonly used ageappropriate assessments of academic achievement, and it is used as an indication of a child's cognitive development. This illustrative example has the following main objectives: (1) investigate whether the trajectories of PIAT scores in mathematics contain heterogeneous patterns, (2) examine how "gender" and "home environment" influence the latent class membership, and (3) examine how "behavior problems" influences the developmental trend of PIAT scores in each trajectory class. A path diagram of the proposed GLCMM is depicted in Figure 7. The covariate "gender, $b$ ", with "1" representing female, and an explanatory latent variable "home environment, $\zeta$ " were used to model the class probability via the multinomial logit model (13). Three observed indicators, "the household variable, $v_{1}$ ", "the score of home cognitive stimulation, $v_{2}$ ", and "emotional support, $v_{3}$ ", were used to measure the latent variable "home environment, $\zeta$ " through Model (19). Among these indicators, $v_{1}$ is a binary variable coded with $\{0,1\}$ representing "in household with father and other relatives" and "in household with mother," respectively, whereas $v_{2}$ and $v_{3}$ are continuous variables. The within-class LCMs are defined by Models (3), (6), and $(8)$, in which the explanatory latent variable "behavior problems, $\xi$ " was measured by five BPI subscales for antisocial $\left(x_{1}\right)$, anxious $\left(x_{2}\right)$, peer conflict $\left(x_{3}\right)$, headstrong $\left(x_{4}\right)$, 
Table 2. Performance of the Bayesian estimates in the simulation study with Type II prior input under sample size $n=800$

\begin{tabular}{|c|c|c|c|c|c|c|c|c|c|c|c|c|c|c|}
\hline & & Class 1 & & & Class 2 & & & & & Class 1 & & & Class 2 & \\
\hline Par & True & Bias & RMS & True & Bias & RMS & & Par & True & Bias & RMS & True & Bias & RMS \\
\hline$\overline{\lambda_{21}}$ & 0.8 & 0.011 & 0.044 & 0.4 & 0.017 & 0.039 & & $\psi_{y 5}$ & 0.36 & 0.007 & 0.035 & 0.25 & 0.025 & 0.036 \\
\hline$\lambda_{31}$ & 0.8 & 0.018 & 0.049 & 0.4 & 0.018 & 0.039 & & $\psi_{y 6}$ & 0.36 & 0.011 & 0.032 & 0.25 & 0.014 & 0.027 \\
\hline$\lambda_{52}$ & 0.8 & 0.010 & 0.046 & 0.4 & 0.010 & 0.043 & & $\psi_{y 7}$ & 0.36 & 0.001 & 0.037 & 0.25 & 0.009 & 0.028 \\
\hline$\lambda_{62}$ & 0.8 & 0.010 & 0.045 & 0.4 & 0.019 & 0.040 & & $\psi_{y 8}$ & 0.36 & 0.023 & 0.059 & 0.25 & 0.045 & 0.054 \\
\hline$\mu_{1}$ & 2.0 & -0.032 & 0.158 & 1.0 & 0.064 & 0.162 & & $\psi_{x 1}$ & 0.36 & 0.065 & 0.091 & 0.25 & 0.048 & 0.059 \\
\hline$\mu_{2}$ & 1.0 & 0.017 & 0.167 & 1.5 & 0.250 & 0.324 & & $\psi_{x 2}$ & 0.36 & 0.008 & 0.028 & 0.25 & 0.007 & 0.020 \\
\hline$\mu_{3}$ & -2.0 & -0.024 & 0.053 & 1.5 & 0.009 & 0.043 & & $\psi_{x 3}$ & 0.36 & 0.012 & 0.035 & 0.25 & 0.007 & 0.024 \\
\hline$\mu_{4}$ & -1.5 & -0.047 & 0.062 & 0.5 & 0.008 & 0.044 & & $\psi_{x 4}$ & 0.36 & 0.069 & 0.098 & 0.25 & 0.037 & 0.050 \\
\hline$a_{11}$ & 0.5 & -0.034 & 0.135 & -0.5 & -0.044 & 0.147 & & $\psi_{x 5}$ & 0.36 & 0.013 & 0.031 & 0.25 & 0.011 & 0.022 \\
\hline$a_{21}$ & -0.5 & -0.011 & 0.097 & 0.5 & 0.064 & 0.138 & & $\psi_{x 6}$ & 0.36 & 0.005 & 0.030 & 0.25 & 0.006 & 0.019 \\
\hline$a_{31}$ & 0.5 & -0.010 & 0.043 & -0.5 & 0.001 & 0.046 & & $\psi_{\delta 1}$ & 0.36 & 0.176 & 0.215 & 0.25 & 0.175 & 0.180 \\
\hline$a_{41}$ & -0.5 & 0.002 & 0.031 & 0.5 & -0.001 & 0.036 & & $\psi_{\delta 2}$ & 0.36 & 0.172 & 0.210 & 0.25 & 0.147 & 0.162 \\
\hline$\gamma_{11}$ & 0.5 & -0.022 & 0.186 & -0.5 & -0.051 & 0.229 & & $\psi_{\delta 3}$ & 0.36 & 0.020 & 0.054 & 0.25 & 0.022 & 0.040 \\
\hline$\gamma_{12}$ & 0.5 & -0.016 & 0.203 & -0.5 & -0.054 & 0.190 & & $\psi_{\delta 4}$ & 0.36 & 0.035 & 0.052 & 0.25 & 0.006 & 0.024 \\
\hline$\gamma_{13}$ & 0.5 & 0.144 & 0.231 & -0.5 & -0.069 & 0.192 & & $\phi_{11}$ & 1.0 & -0.027 & 0.101 & 1.0 & -0.084 & 0.123 \\
\hline$\gamma_{21}$ & 0.5 & -0.014 & 0.174 & -0.5 & -0.103 & 0.253 & & $\phi_{21}$ & 0.3 & -0.004 & 0.048 & 0.3 & -0.052 & 0.081 \\
\hline$\gamma_{22}$ & 0.5 & -0.012 & 0.167 & -0.5 & -0.107 & 0.233 & & $\phi_{22}$ & 1.0 & -0.014 & 0.091 & 1.0 & -0.079 & 0.125 \\
\hline$\gamma_{23}$ & 0.5 & 0.069 & 0.208 & -0.5 & -0.018 & 0.186 & & $\varphi_{0}$ & -0.5 & 0.044 & 0.130 & $0.0^{*}$ & & \\
\hline$\gamma_{31}$ & 0.5 & 0.011 & 0.062 & -0.5 & -0.004 & 0.058 & & $\varphi_{1}$ & 1.0 & -0.014 & 0.099 & $0.0^{*}$ & & \\
\hline$\gamma_{32}$ & 0.5 & -0.004 & 0.060 & -0.5 & 0.012 & 0.053 & & $\varphi_{2}$ & 0.8 & 0.026 & 0.119 & $0.0^{*}$ & & \\
\hline$\gamma_{33}$ & 0.5 & 0.050 & 0.077 & -0.5 & -0.011 & 0.077 & & $\varphi_{3}$ & 0.8 & 0.000 & 0.105 & $0.0^{*}$ & & \\
\hline$\gamma_{41}$ & 0.5 & 0.000 & 0.054 & -0.5 & 0.006 & 0.047 & & & & & & & & \\
\hline$\gamma_{42}$ & 0.5 & 0.005 & 0.069 & -0.5 & 0.000 & 0.049 & & & & & & & & \\
\hline$\gamma_{43}$ & 0.5 & 0.075 & 0.095 & -0.5 & -0.010 & 0.068 & & & & & & & & \\
\hline$\overline{\mathrm{Par}}$ & True & Bias & RMS & Par & True & Bias & RMS & & & & & & & \\
\hline$\overline{\lambda_{v, 21}}$ & 0.8 & 0.004 & 0.034 & $\psi_{v 1}$ & 0.36 & 0.004 & 0.034 & & & & & & & \\
\hline$\lambda_{v, 31}$ & 0.8 & 0.004 & 0.038 & $\psi_{v 2}$ & 0.36 & 0.003 & 0.024 & & & & & & & \\
\hline$\lambda_{v, 52}$ & 0.8 & 0.010 & 0.035 & $\psi_{v 3}$ & 0.36 & 0.000 & 0.024 & & & & & & & \\
\hline$\lambda_{v, 62}$ & 0.8 & 0.004 & 0.034 & $\psi_{v 4}$ & 0.36 & 0.010 & 0.033 & & & & & & & \\
\hline$\phi_{v, 11}$ & 1.0 & 0.004 & 0.080 & $\psi_{v 5}$ & 0.36 & 0.000 & 0.020 & & & & & & & \\
\hline$\phi_{v, 21}$ & 0.3 & -0.002 & 0.041 & $\psi_{v 6}$ & 0.36 & 0.004 & 0.025 & & & & & & & \\
\hline$\phi_{v, 22}$ & 1.0 & 0.002 & 0.076 & & & & & & & & & & & \\
\hline
\end{tabular}

Note: The subscript $k$ is suppressed for notational simplicity, and the 0's with asterisk are fixed for identification purpose.

and hyperactive behaviors $\left(x_{5}\right)$. Higher scores represent a greater level of behavior problems. The scores used in this study were normed; thus, they can be treated as continuous. Given that the values of the continuous variables were quite large, the longitudinal measurements $y_{1}$ to $y_{3}$ were divided by 10 for readability, and the continuous observed indicators $v_{2}, v_{3}$, and $x_{1}$ to $x_{5}$ were standardized before analysis. The proposed GLCMM was used to fit the data set that involves both continuous and binary data.

The modified DIC was used to determine the number of trajectory classes, $K$. Let $M_{k}$ denote the $k$-class model, $k=1, \ldots, 5$. Given that we had little information about the model and the parameters, we assigned the vague prior inputs in (20) as follows: elements in $\left\{\boldsymbol{\mu}_{0 k}, \boldsymbol{\Lambda}_{0 \epsilon k m}, \boldsymbol{\Lambda}_{0 \delta k l}\right.$, $\left.\boldsymbol{\varphi}_{0 k}, \boldsymbol{\Lambda}_{0 v l}\right\}$ were fixed at $0.0, \alpha_{0 \epsilon k m}=\alpha_{0 \delta k l}=9, \beta_{0 \epsilon k m}=$ $\beta_{0 \delta k l}=4, \boldsymbol{\Sigma}_{0 k}, \mathbf{H}_{0 \epsilon k m}, \mathbf{H}_{0 \delta k l}, \boldsymbol{\Sigma}_{\varphi 0 k} ; \mathbf{H}_{0 v l}$ were identity matrices with appropriate orders, $\rho_{0}=\rho_{0 v}=5, \mathbf{R}_{0 k}^{-1}$ and $\mathbf{R}_{0 v}^{-1}$ were diagonal matrices with the diagonal elements 2.0. The values of the modified DICs were $D I C_{M_{1}}=51,883$,
$D I C_{M_{2}}=51,352, D I C_{M_{3}}=52,088, D I C_{M_{4}}=53,835$, and $D I C_{M_{5}}=55,406$. Therefore, the 2-class model, $M_{2}$, was selected.

Based on the random permutation sampler, we found that $\mu_{11}<\mu_{21}$ is an appropriate identifiability constraint. Furthermore, our pilot study showed that the MCMC algorithm converged within 20,000 iterations. Figure 8 shows the plot of the EPSR values against the iteration numbers. After discarding 20,000 burn-in iterations, we collected additional 20,000 observation to obtain the Bayesian estimates (EST) of the unknown parameters and their corresponding standard error estimates (SE). Table 6 summarizes the results. The main findings are as follows. First, more than one latent trajectory class exist, each of which has a different trajectory pattern. On average, the children in Class 1 have low initial PIAT scores in mathematics but a high rate of change across time, whereas those in Class 2 present an opposite pattern-high initial scores but low rate of change. In practice, there might be more classes of students, for example, 
Table 3. Performance of the Bayesian estimates in the simulation study with Type I prior input under sample size $n=1,600$

\begin{tabular}{|c|c|c|c|c|c|c|c|c|c|c|c|c|c|c|}
\hline & & Class 1 & & & Class 2 & & & & & Class 1 & & & Class 2 & \\
\hline$\overline{\mathrm{Par}}$ & True & Bias & RMS & True & Bias & RMS & & Par & True & Bias & RMS & True & Bias & RMS \\
\hline$\overline{\lambda_{21}}$ & 0.8 & 0.012 & 0.037 & 0.4 & 0.015 & 0.031 & & $\psi_{y 5}$ & 0.36 & 0.008 & 0.028 & 0.25 & 0.012 & 0.027 \\
\hline$\lambda_{31}$ & 0.8 & 0.014 & 0.033 & 0.4 & 0.021 & 0.035 & & $\psi_{y 6}$ & 0.36 & 0.001 & 0.019 & 0.25 & 0.009 & 0.019 \\
\hline$\lambda_{52}$ & 0.8 & 0.013 & 0.032 & 0.4 & 0.015 & 0.030 & & $\psi_{y 7}$ & 0.36 & 0.002 & 0.024 & 0.25 & 0.006 & 0.022 \\
\hline$\lambda_{62}$ & 0.8 & 0.009 & 0.030 & 0.4 & 0.016 & 0.030 & & $\psi_{y 8}$ & 0.36 & 0.021 & 0.046 & 0.25 & 0.024 & 0.037 \\
\hline$\mu_{1}$ & 2.0 & 0.004 & 0.131 & 1.0 & 0.047 & 0.118 & & $\psi_{x 1}$ & 0.36 & 0.034 & 0.048 & 0.25 & 0.045 & 0.059 \\
\hline$\mu_{2}$ & 1.0 & 0.036 & 0.154 & 1.5 & 0.181 & 0.231 & & $\psi_{x 2}$ & 0.36 & 0.002 & 0.021 & 0.25 & 0.007 & 0.015 \\
\hline$\mu_{3}$ & -2.0 & -0.015 & 0.038 & 1.5 & 0.017 & 0.043 & & $\psi_{x 3}$ & 0.36 & 0.007 & 0.022 & 0.25 & 0.003 & 0.015 \\
\hline$\mu_{4}$ & -1.5 & -0.020 & 0.034 & 0.5 & 0.012 & 0.031 & & $\psi_{x 4}$ & 0.36 & 0.036 & 0.053 & 0.25 & 0.044 & 0.058 \\
\hline$a_{11}$ & 0.5 & -0.003 & 0.107 & -0.5 & -0.023 & 0.105 & & $\psi_{x 5}$ & 0.36 & 0.000 & 0.021 & 0.25 & 0.005 & 0.016 \\
\hline$a_{21}$ & -0.5 & -0.015 & 0.070 & 0.5 & 0.041 & 0.099 & & $\psi_{x 6}$ & 0.36 & 0.004 & 0.020 & 0.25 & 0.007 & 0.016 \\
\hline$a_{31}$ & 0.5 & -0.004 & 0.027 & -0.5 & 0.002 & 0.027 & & $\psi_{\delta 1}$ & 0.36 & 0.172 & 0.212 & 0.25 & 0.165 & 0.179 \\
\hline$a_{41}$ & -0.5 & 0.000 & 0.027 & 0.5 & -0.005 & 0.026 & & $\psi_{\delta 2}$ & 0.36 & 0.119 & 0.171 & 0.25 & 0.125 & 0.140 \\
\hline$\gamma_{11}$ & 0.5 & 0.007 & 0.136 & -0.5 & -0.038 & 0.132 & & $\psi_{\delta 3}$ & 0.36 & 0.006 & 0.033 & 0.25 & 0.013 & 0.030 \\
\hline$\gamma_{12}$ & 0.5 & 0.010 & 0.141 & -0.5 & -0.045 & 0.130 & & $\psi_{\delta 4}$ & 0.36 & 0.015 & 0.032 & 0.25 & 0.009 & 0.023 \\
\hline$\gamma_{13}$ & 0.5 & 0.106 & 0.201 & -0.5 & -0.005 & 0.154 & & $\phi_{11}$ & 1.0 & -0.022 & 0.078 & 1.0 & -0.062 & 0.098 \\
\hline$\gamma_{21}$ & 0.5 & -0.006 & 0.121 & -0.5 & -0.091 & 0.148 & & $\phi_{21}$ & 0.3 & -0.008 & 0.042 & 0.3 & -0.021 & 0.051 \\
\hline$\gamma_{22}$ & 0.5 & -0.001 & 0.117 & -0.5 & -0.076 & 0.162 & & $\phi_{22}$ & 1.0 & -0.014 & 0.064 & 1.0 & -0.064 & 0.103 \\
\hline$\gamma_{23}$ & 0.5 & 0.042 & 0.139 & -0.5 & -0.047 & 0.155 & & $\varphi_{0}$ & -0.5 & 0.015 & 0.094 & $0.0^{*}$ & & \\
\hline$\gamma_{31}$ & 0.5 & 0.003 & 0.041 & -0.5 & -0.004 & 0.040 & & $\varphi_{1}$ & 1.0 & 0.003 & 0.078 & $0.0^{*}$ & & \\
\hline$\gamma_{32}$ & 0.5 & 0.000 & 0.040 & -0.5 & -0.003 & 0.041 & & $\varphi_{2}$ & 0.8 & 0.010 & 0.090 & $0.0^{*}$ & & \\
\hline$\gamma_{33}$ & 0.5 & 0.029 & 0.047 & -0.5 & -0.061 & 0.103 & & $\varphi_{3}$ & 0.8 & 0.002 & 0.089 & $0.0^{*}$ & & \\
\hline$\gamma_{41}$ & 0.5 & -0.001 & 0.039 & -0.5 & -0.009 & 0.040 & & & & & & & & \\
\hline$\gamma_{42}$ & 0.5 & -0.002 & 0.036 & -0.5 & -0.004 & 0.044 & & & & & & & & \\
\hline$\gamma_{43}$ & 0.5 & 0.029 & 0.045 & -0.5 & -0.052 & 0.083 & & & & & & & & \\
\hline$\overline{\overline{\mathrm{Par}}}$ & True & Bias & RMS & Par & True & Bias & RMS & & & & & & & \\
\hline$\overline{\lambda_{v, 21}}$ & 0.8 & -0.003 & 0.023 & $\psi_{v 1}$ & 0.36 & 0.004 & 0.026 & & & & & & & \\
\hline$\lambda_{v, 31}$ & 0.8 & 0.000 & 0.024 & $\psi_{v 2}$ & 0.36 & 0.006 & 0.018 & & & & & & & \\
\hline$\lambda_{v, 52}$ & 0.8 & 0.001 & 0.023 & $\psi_{v 3}$ & 0.36 & 0.001 & 0.020 & & & & & & & \\
\hline$\lambda_{v, 62}$ & 0.8 & -0.001 & 0.025 & $\psi_{v 4}$ & 0.36 & 0.004 & 0.023 & & & & & & & \\
\hline$\phi_{v, 11}$ & 1.0 & 0.010 & 0.049 & $\psi_{v 5}$ & 0.36 & 0.004 & 0.020 & & & & & & & \\
\hline$\phi_{v, 21}$ & 0.3 & 0.005 & 0.027 & $\psi_{v 6}$ & 0.36 & 0.003 & 0.016 & & & & & & & \\
\hline$\phi_{v, 22}$ & 1.0 & 0.002 & 0.052 & & & & & & & & & & & \\
\hline
\end{tabular}

Note: The subscript $k$ is suppressed for notational simplicity, and the 0's with asterisk are fixed for identification purpose.

students with low (high) initial PIAT scores in mathematics also have low (high) rates of change across time. However, we did not identify such classes of students in the NLSY data. Second, the influence of "behavior problems" on the latent growth factors is significant in Class 2 but not in Class 1. Third, in the multinomial logit model, $\varphi_{1}$ and $\varphi_{2}$ are significantly positive, indicating that girls and children with a better "home environment" are more likely to belong to Class 1. Finally, some of the other parameters, including $\lambda$ 's, $\psi_{\epsilon}$ 's, $\phi$ 's, and $\psi_{\delta}$ 's, vary substantially across classes. This finding confirms the existence of heterogeneity within the NLSY data.

Using a single PC with an Intel Core i3 CPU 550@3.20 $\mathrm{GHz}$ and $4.00 \mathrm{~GB}$ RAM, the computing time for obtaining the Bayesian estimates of parameters and the modified DIC value under $M_{2}$ was approximately 100 minutes. Our program is written in $\mathrm{C}$ language and is available upon request.

To assess the sensitivity of the Bayesian estimation and model selection to different prior inputs, the above analysis was repeated with some disturbances to the current prior input. Still, the 2-class model was selected, and the Bayesian estimates of the unknown parameters under the selected model were close to those presented in Table 6 . The results are not reported here.

\section{DISCUSSION}

In this article, an integrated generalized mixture LCM was established under the Bayesian framework. Unlike traditional LCMs, this integrated model can simultaneously handle different kinds of latent and observed variables, such as (1) continuous latent variables, including latent growth factors and explanatory latent variables; (2) a latent allocation variable representing latent trajectory classes; (3) fixed and latent effects in predicting latent growth factors and probabilities of subjects' class memberships; and (4) mixed continuous and discrete observed variables that follow the EFD. The proposed GLCMM represents a broad class of statistical models flexible enough to detect the heterogeneity of a longitudinal trajectory pattern, explore fixed and latent effects that influence both longitudinal change patterns 
Table 4. Performance of the Bayesian estimates in the simulation study with Type II prior input under sample size $n=1,600$

\begin{tabular}{|c|c|c|c|c|c|c|c|c|c|c|c|c|c|c|}
\hline & & Class 1 & & & Class & & & & & Class 1 & & & Class 2 & \\
\hline Par & True & Bias & RMS & True & Bias & RMS & & Par & True & Bias & RMS & True & Bias & RMS \\
\hline$\overline{\lambda_{21}}$ & 0.8 & 0.007 & 0.036 & 0.4 & 0.011 & 0.028 & & $\psi_{y 5}$ & 0.36 & 0.004 & 0.032 & 0.25 & 0.004 & 0.022 \\
\hline$\lambda_{31}$ & 0.8 & 0.008 & 0.030 & 0.4 & 0.012 & 0.027 & & $\psi_{y 6}$ & 0.36 & -0.003 & 0.021 & 0.25 & 0.002 & 0.015 \\
\hline$\lambda_{52}$ & 0.8 & 0.013 & 0.036 & 0.4 & 0.005 & 0.030 & & $\psi_{y 7}$ & 0.36 & 0.003 & 0.024 & 0.25 & 0.001 & 0.018 \\
\hline$\lambda_{62}$ & 0.8 & 0.013 & 0.032 & 0.4 & 0.004 & 0.027 & & $\psi_{y 8}$ & 0.36 & -0.002 & 0.043 & 0.25 & 0.008 & 0.036 \\
\hline$\mu_{1}$ & 2.0 & -0.028 & 0.112 & 1.0 & 0.030 & 0.109 & & $\psi_{x 1}$ & 0.36 & 0.036 & 0.056 & 0.25 & 0.019 & 0.033 \\
\hline$\mu_{2}$ & 1.0 & -0.029 & 0.138 & 1.5 & 0.123 & 0.185 & & $\psi_{x 2}$ & 0.36 & 0.004 & 0.022 & 0.25 & -0.001 & 0.014 \\
\hline$\mu_{3}$ & -2.0 & -0.010 & 0.030 & 1.5 & 0.008 & 0.031 & & $\psi_{x 3}$ & 0.36 & 0.003 & 0.022 & 0.25 & 0.002 & 0.013 \\
\hline$\mu_{4}$ & -1.5 & -0.025 & 0.037 & 0.5 & -0.001 & 0.026 & & $\psi_{x 4}$ & 0.36 & 0.035 & 0.060 & 0.25 & 0.013 & 0.034 \\
\hline$a_{11}$ & 0.5 & -0.035 & 0.102 & -0.5 & -0.011 & 0.114 & & $\psi_{x 5}$ & 0.36 & 0.002 & 0.026 & 0.25 & 0.000 & 0.017 \\
\hline$a_{21}$ & -0.5 & 0.005 & 0.066 & 0.5 & 0.006 & 0.072 & & $\psi_{x 6}$ & 0.36 & 0.004 & 0.022 & 0.25 & 0.002 & 0.017 \\
\hline$a_{31}$ & 0.5 & -0.005 & 0.028 & -0.5 & -0.001 & 0.030 & & $\psi_{\delta 1}$ & 0.36 & 0.049 & 0.156 & 0.25 & 0.046 & 0.060 \\
\hline$a_{41}$ & -0.5 & 0.006 & 0.029 & 0.5 & 0.002 & 0.022 & & $\psi_{\delta 2}$ & 0.36 & 0.052 & 0.123 & 0.25 & 0.047 & 0.077 \\
\hline$\gamma_{11}$ & 0.5 & -0.011 & 0.139 & -0.5 & -0.002 & 0.128 & & $\psi_{\delta 3}$ & 0.36 & 0.004 & 0.036 & 0.25 & 0.006 & 0.027 \\
\hline$\gamma_{12}$ & 0.5 & -0.007 & 0.143 & -0.5 & -0.001 & 0.143 & & $\psi_{\delta 4}$ & 0.36 & 0.018 & 0.035 & 0.25 & 0.005 & 0.023 \\
\hline$\gamma_{13}$ & 0.5 & 0.077 & 0.174 & -0.5 & -0.013 & 0.134 & & $\phi_{11}$ & 1.0 & -0.024 & 0.075 & 1.0 & -0.041 & 0.089 \\
\hline$\gamma_{21}$ & 0.5 & -0.028 & 0.114 & -0.5 & -0.074 & 0.145 & & $\phi_{21}$ & 0.3 & -0.022 & 0.048 & 0.3 & -0.029 & 0.055 \\
\hline$\gamma_{22}$ & 0.5 & -0.027 & 0.116 & -0.5 & -0.051 & 0.142 & & $\phi_{22}$ & 1.0 & -0.025 & 0.074 & 1.0 & -0.031 & 0.085 \\
\hline$\gamma_{23}$ & 0.5 & 0.027 & 0.140 & -0.5 & -0.030 & 0.153 & & $\varphi_{0}$ & -0.5 & 0.013 & 0.091 & $0.0^{*}$ & & \\
\hline$\gamma_{31}$ & 0.5 & 0.003 & 0.041 & -0.5 & -0.008 & 0.039 & & $\varphi_{1}$ & 1.0 & 0.006 & 0.075 & $0.0^{*}$ & & \\
\hline$\gamma_{32}$ & 0.5 & 0.003 & 0.037 & -0.5 & 0.009 & 0.037 & & $\varphi_{2}$ & 0.8 & 0.007 & 0.082 & $0.0^{*}$ & & \\
\hline$\gamma_{33}$ & 0.5 & 0.024 & 0.051 & -0.5 & -0.002 & 0.041 & & $\varphi_{3}$ & 0.8 & -0.010 & 0.087 & $0.0^{*}$ & & \\
\hline$\gamma_{41}$ & 0.5 & -0.008 & 0.039 & -0.5 & 0.001 & 0.039 & & & & & & & & \\
\hline$\gamma_{42}$ & 0.5 & 0.006 & 0.041 & -0.5 & 0.006 & 0.036 & & & & & & & & \\
\hline$\gamma_{43}$ & 0.5 & 0.044 & 0.071 & -0.5 & -0.001 & 0.035 & & & & & & & & \\
\hline$\overline{\mathrm{Par}}$ & True & Bias & RMS & Par & True & Bias & RMS & & & & & & & \\
\hline$\overline{\lambda_{v, 21}}$ & 0.8 & -0.002 & 0.022 & $\psi_{v 1}$ & 0.36 & 0.002 & 0.024 & & & & & & & \\
\hline$\lambda_{v, 31}$ & 0.8 & -0.001 & 0.022 & $\psi_{v 2}$ & 0.36 & 0.000 & 0.017 & & & & & & & \\
\hline$\lambda_{v, 52}$ & 0.8 & -0.001 & 0.024 & $\psi_{v 3}$ & 0.36 & 0.005 & 0.019 & & & & & & & \\
\hline$\lambda_{v, 62}$ & 0.8 & 0.001 & 0.022 & $\psi_{v 4}$ & 0.36 & -0.003 & 0.024 & & & & & & & \\
\hline$\phi_{v, 11}$ & 1.0 & 0.004 & 0.050 & $\psi_{v 5}$ & 0.36 & 0.001 & 0.019 & & & & & & & \\
\hline$\phi_{v, 21}$ & 0.3 & -0.007 & 0.030 & $\psi_{v 6}$ & 0.36 & 0.001 & 0.017 & & & & & & & \\
\hline$\phi_{v, 22}$ & 1.0 & 0.004 & 0.049 & & & & & & & & & & & \\
\hline
\end{tabular}

Note: The subscript $k$ is suppressed for notational simplicity, and the 0's with asterisk are fixed for identification purpose.

Table 5. Performance of the modified DIC in model selection with Type I and Type II prior inputs under sample sizes $n=800$ and $n=1,600$ (the presented values are means and standard deviations)

\begin{tabular}{rrrrr}
\hline \hline & & & & \multicolumn{1}{c}{$n=1,600$} \\
\hline & \multicolumn{1}{c}{ Prior I } & Prior II & \multicolumn{1}{c}{ Prior I } & \multicolumn{1}{c}{ Prior II } \\
\hline$M_{1}$ & $50410.99(340.27)$ & $50029.04(415.41)$ & $101261.54(545.98)$ & $100995.04(584.94)$ \\
$M_{2}$ & $45979.87(267.76)$ & $45131.99(315.20)$ & $91574.05(470.69)$ & $90331.27(490.70)$ \\
$M_{3}$ & $47182.20(1022.62)$ & $48237.50(2642.64)$ & $100469.55(6369.87)$ & $99088.84(7086.21)$ \\
\hline
\end{tabular}

and latent class membership, and accommodate mixed-data types. Our proposed Bayesian approach, which includes the Gibbs sampler, the $\mathrm{MH}$ algorithm, the permutation sampler, and the modified DIC, solves computational challenges that arise from the GLCMM modeling efforts. The NLSY example demonstrates how the proposed GLCMM can be applied to longitudinal data and how it can test hypothesis outcomes that are generally not achievable using conventional methods.

The proposed GLCMM can be extended to several directions. First, our model assumes that data are collected with the same number of waves and the same spacing of waves among individuals (i.e., balanced data). However, unbalanced setting is common in longitudinal data. Hence, extending the current model to deal with unbalanced data is worthy of further consideration. Second, the proposed model can be extended to include longitudinal latent effects to predict latent growth factors and the probability of class membership. Third, given that ordered and unordered categorical data are very common in practical research, the idea of probit (logit) regression or cumulative probit (logit) regression methods $[1,4,33,36]$ could be applied to incorporate these 


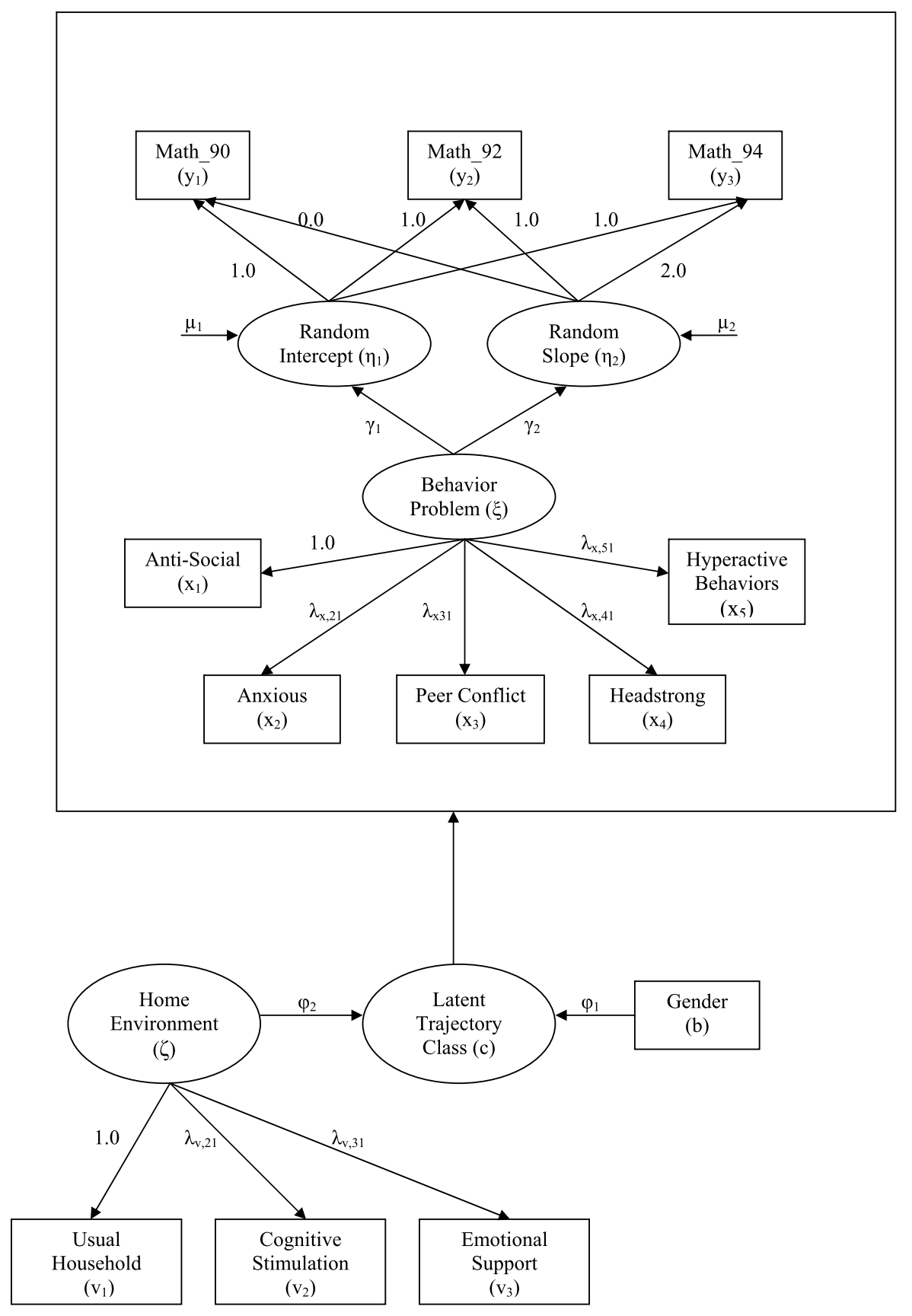

Figure 7. Path diagram of proposed model in the illustrative example.

types of data in the current model. Finally, except for determining the number of latent trajectory classes, the modified DIC can also be used to select appropriate linear or nonlinear parametric functions $f_{1}, \ldots, f_{h}$. Moreover, the parametric trajectory equation and parametric functions $f_{1}, \ldots, f_{h}$ in the current model can be further extended to nonparametric forms. The Bayesian P-splines approach [13, 34, 35] is a promising candidate for the nonparametric modeling of LCMs.

\section{APPENDIX A. FULL CONDITIONAL DISTRIBUTIONS}

(a) The full conditional distribution of $\varphi_{k}$ :

$$
\begin{aligned}
& p\left(\boldsymbol{\varphi}_{k} \mid \mathbf{U}, \mathbf{V}, \boldsymbol{\Omega}, \widetilde{\boldsymbol{\Omega}}, \mathbf{C}, \boldsymbol{\theta}\right) \\
& \quad \propto \prod_{i=1}^{n} p\left(c_{i} \mid \boldsymbol{\varphi}_{k}, \boldsymbol{\theta}, \mathbf{u}_{i}, \mathbf{v}_{i}, \boldsymbol{\omega}_{i}, \boldsymbol{\zeta}_{i}\right) p\left(\boldsymbol{\varphi}_{k}\right)
\end{aligned}
$$


Table 6. Bayesian estimates and their corresponding standard error estimates in the illustrative example

\begin{tabular}{|c|c|c|c|c|}
\hline \multirow{2}{*}{ Par } & \multicolumn{2}{|c|}{ Class 1} & \multicolumn{2}{|c|}{ Class 2} \\
\hline & EST & $\mathrm{SE}$ & EST & $\mathrm{SE}$ \\
\hline$\overline{\mu_{1}}$ & 2.613 & 0.036 & 4.321 & 0.209 \\
\hline$\mu_{2}$ & 1.076 & 0.013 & 0.462 & 0.104 \\
\hline$\gamma_{1}$ & -0.058 & 0.061 & -0.687 & 0.147 \\
\hline$\gamma_{2}$ & -0.042 & 0.026 & 0.192 & 0.068 \\
\hline$\lambda_{x 21}$ & 0.926 & 0.051 & 0.889 & 0.095 \\
\hline$\lambda_{x 31}$ & 1.119 & 0.054 & 0.706 & 0.071 \\
\hline$\lambda_{x 41}$ & 0.988 & 0.049 & 0.738 & 0.071 \\
\hline$\lambda_{x 51}$ & 0.761 & 0.046 & 1.219 & 0.128 \\
\hline$\phi_{x}$ & 0.435 & 0.034 & 1.987 & 0.456 \\
\hline$\psi_{\epsilon 1}$ & 0.419 & 0.027 & 0.400 & 0.103 \\
\hline$\psi_{\epsilon 2}$ & 0.336 & 0.021 & 0.659 & 0.171 \\
\hline$\psi_{\epsilon 3}$ & 0.288 & 0.025 & 0.374 & 0.097 \\
\hline$\psi_{\epsilon 4}$ & 0.457 & 0.026 & 0.766 & 0.187 \\
\hline$\psi_{\epsilon 5}$ & 0.567 & 0.025 & 0.418 & 0.115 \\
\hline$\psi_{\epsilon 6}$ & 0.439 & 0.024 & 0.337 & 0.069 \\
\hline$\psi_{\epsilon 7}$ & 0.554 & 0.025 & 0.307 & 0.061 \\
\hline$\psi_{\epsilon 8}$ & 0.574 & 0.026 & 0.824 & 0.217 \\
\hline$\psi_{\delta 1}$ & 1.035 & 0.049 & 0.674 & 0.170 \\
\hline$\psi_{\delta 2}$ & 0.067 & 0.006 & 0.218 & 0.045 \\
\hline$\varphi_{0}$ & 2.118 & 0.284 & $0.0^{*}$ & \\
\hline$\varphi_{1}$ & 4.321 & 0.209 & $0.0^{*}$ & \\
\hline$\varphi_{2}$ & 0.462 & 0.104 & $0.0^{*}$ & \\
\hline \multicolumn{5}{|c|}{ Par. related to $\zeta$} \\
\hline$\overline{\lambda_{v 21}}$ & 1.525 & 0.259 & & \\
\hline$\lambda_{v 31}$ & 1.273 & 0.243 & & \\
\hline$\phi_{v}$ & 0.114 & 0.025 & & \\
\hline$\psi_{v 1}$ & 0.735 & 0.077 & & \\
\hline$\psi_{v 2}$ & 0.816 & 0.056 & & \\
\hline
\end{tabular}

Note: The subscript $k$ is suppressed for notational simplicity, and the 0's with asterisk are fixed for identification purpose.

$$
\begin{aligned}
\propto & \exp \left\{\sum_{i: c_{i}=k} \boldsymbol{\varphi}_{k}^{T} \mathbf{G}\left(\boldsymbol{\zeta}_{i}\right)-\sum_{i=1}^{n} \log \left[\sum_{j=1}^{K} \exp \left(\boldsymbol{\varphi}_{j}^{T} \mathbf{G}\left(\boldsymbol{\zeta}_{i}\right)\right)\right]\right. \\
& \left.-\frac{1}{2}\left(\boldsymbol{\varphi}_{k}-\boldsymbol{\varphi}_{0 k}\right)^{T} \boldsymbol{\Sigma}_{\varphi 0 k}^{-1}\left(\boldsymbol{\varphi}_{k}-\boldsymbol{\varphi}_{0 k}\right)\right\}
\end{aligned}
$$

where $\mathbf{G}\left(\boldsymbol{\zeta}_{i}\right)=\left(1, \mathbf{b}_{i}^{T}, \boldsymbol{\zeta}_{i}^{T}\right)^{T}$.

(b) The full conditional distribution of $\widetilde{\boldsymbol{\Omega}}$ :

$$
\begin{aligned}
p\left(\boldsymbol{\zeta}_{i} \mid \mathbf{u}_{i}, \mathbf{v}_{i}, \boldsymbol{\omega}_{i}, \mathbf{c}_{i}=k, \boldsymbol{\theta}\right) & \\
\quad & p\left(\mathbf{v}_{i} \mid \boldsymbol{\zeta}_{i}, \boldsymbol{\theta}\right) p\left(c_{i}=k \mid \boldsymbol{\zeta}_{i}, \boldsymbol{\theta}\right) p\left(\boldsymbol{\zeta}_{i} \mid \boldsymbol{\theta}\right) \\
& \propto \exp \left\{-\frac{1}{2}\left(\mathbf{v}_{i}-\boldsymbol{\Lambda}_{v} \boldsymbol{\zeta}_{i}\right)^{T} \boldsymbol{\Psi}_{v}^{-1}\left(\mathbf{v}_{i}-\boldsymbol{\Lambda}_{v} \boldsymbol{\zeta}_{i}\right)\right. \\
& \left.-\frac{1}{2} \boldsymbol{\zeta}_{i}^{T} \boldsymbol{\Phi}_{v}^{-1} \boldsymbol{\zeta}_{i}+\boldsymbol{\varphi}_{k}^{T} \mathbf{G}\left(\boldsymbol{\zeta}_{i}\right)-\log \left[\sum_{j=1}^{K} \exp \left(\boldsymbol{\varphi}_{j}^{T} \mathbf{G}\left(\boldsymbol{\zeta}_{i}\right)\right)\right]\right\} .
\end{aligned}
$$

(c) The full conditional distribution of $\boldsymbol{\Omega}$ :

$$
p(\boldsymbol{\Omega} \mid \mathbf{U}, \mathbf{V}, \mathbf{C}, \widetilde{\boldsymbol{\Omega}}, \boldsymbol{\theta})=\prod_{i=1}^{n} p\left(\boldsymbol{\omega}_{i} \mid \mathbf{u}_{i}, \mathbf{v}_{i}, \mathbf{c}_{i}, \boldsymbol{\zeta}_{i}, \boldsymbol{\theta}\right), \text { and }
$$$$
p\left(\boldsymbol{\omega}_{i} \mid \mathbf{u}_{i}, \mathbf{v}_{i}, \mathbf{c}_{i}, \boldsymbol{\zeta}_{i}, \boldsymbol{\theta}\right)
$$

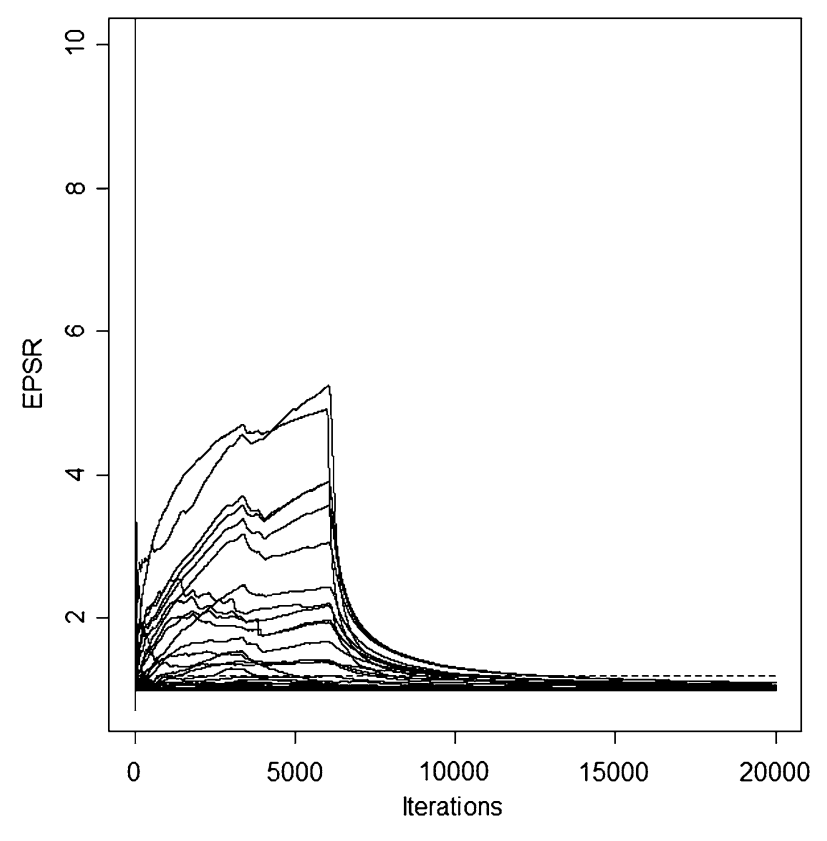

Figure 8 . Plot of the estimated potential scale reduction values against the iteration numbers in the illustrative example.

$$
\begin{aligned}
\propto & \exp \left\{\sum_{j=1}^{p}\left[u_{i j} \vartheta_{k i j}-b_{j}\left(\vartheta_{k i j}\right)\right] / \psi_{\epsilon k j}-\frac{1}{2} \boldsymbol{\xi}_{i}^{T} \boldsymbol{\Phi}_{k}^{-1} \boldsymbol{\xi}_{i}\right. \\
& -\frac{1}{2}\left(\boldsymbol{\eta}_{i}-\boldsymbol{\mu}_{k}-\boldsymbol{\Lambda}_{\delta k} \mathbf{G}\left(\boldsymbol{\omega}_{i}\right)\right)^{T} \\
& \left.\times \boldsymbol{\Psi}_{\delta k}^{-1}\left(\boldsymbol{\eta}_{i}-\boldsymbol{\mu}_{k}-\boldsymbol{\Lambda}_{\delta k} \mathbf{G}\left(\boldsymbol{\omega}_{i}\right)\right)\right\},
\end{aligned}
$$

where $\boldsymbol{\Lambda}_{\delta k}=\left(\mathbf{A}_{k}, \boldsymbol{\Pi}_{k}, \boldsymbol{\Gamma}_{k}\right), \mathbf{G}\left(\boldsymbol{\omega}_{i}\right)=\left(\mathbf{w}_{i}^{T}, \boldsymbol{\eta}_{i}^{T}, \mathbf{F}\left(\boldsymbol{\xi}_{i}\right)^{T}\right)^{T}$.

(d) The full conditional distribution of $\mathbf{C}$ :

$p(\mathbf{C} \mid \mathbf{U}, \mathbf{V}, \boldsymbol{\Omega}, \widetilde{\boldsymbol{\Omega}}, \boldsymbol{\theta})=\prod_{i=1}^{n} p\left(c_{i} \mid \mathbf{u}_{i}, \mathbf{v}_{i}, \boldsymbol{\omega}_{i}, \boldsymbol{\zeta}_{i}, \boldsymbol{\theta}\right)$, and

$p\left(c_{i}=k \mid \mathbf{u}_{i}, \mathbf{v}_{i}, \boldsymbol{\omega}_{i}, \boldsymbol{\zeta}_{i}, \boldsymbol{\theta}\right)$

$=\frac{p\left(\mathbf{u}_{i} \mid c_{i}=k, \boldsymbol{\omega}_{i}, \boldsymbol{\theta}\right) p\left(\boldsymbol{\eta}_{i} \mid c_{i}=k, \boldsymbol{\xi}_{i}, \boldsymbol{\theta}\right) p\left(\boldsymbol{\xi}_{i} \mid c_{i}=k, \boldsymbol{\theta}\right) p\left(c_{i}=k \mid \boldsymbol{\zeta}_{i}, \boldsymbol{\theta}\right)}{\sum_{j=1}^{K} p\left(\mathbf{u}_{i} \mid c_{i}=j, \boldsymbol{\omega}_{i}, \boldsymbol{\theta}\right) p\left(\boldsymbol{\eta}_{i} \mid c_{i}=j, \boldsymbol{\xi}_{i}, \boldsymbol{\theta}\right) p\left(\boldsymbol{\xi}_{i} \mid c_{i}=j, \boldsymbol{\theta}\right) p\left(c_{i}=j \mid \boldsymbol{\zeta}_{i}, \boldsymbol{\theta}\right)}$.

(e) The full conditional distribution of $\boldsymbol{\Lambda}_{k j}$ : for $j=1, \ldots, p$,

$$
\begin{aligned}
& p\left(\boldsymbol{\Lambda}_{k j} \mid \mathbf{U}, \mathbf{V}, \boldsymbol{\Omega}, \widetilde{\boldsymbol{\Omega}}, \mathbf{C}, \boldsymbol{\theta}\right) \\
& \propto \exp \left\{-\frac{1}{2} \psi_{\epsilon k j}^{-1}\left(\boldsymbol{\Lambda}_{k j}-\boldsymbol{\Lambda}_{0 \epsilon k j}\right)^{T} \mathbf{H}_{0 \epsilon k j}^{-1}\left(\boldsymbol{\Lambda}_{k j}-\boldsymbol{\Lambda}_{0 \epsilon k j}\right)\right. \\
& \left.\quad+\sum_{i: c_{i}=k}\left[u_{i j} \vartheta_{k i j}-b_{j}\left(\vartheta_{k i j}\right)\right] / \psi_{\epsilon k j}\right\} .
\end{aligned}
$$

The full conditional distributions of other parameters in $\boldsymbol{\theta}$ are the normal, Gamma, and inverted Wishart distributions. They are not presented here.

Received 27 April 2012 


\section{REFERENCES}

[1] Agresti, A. (2002). Categorical Data Analysis. New Jersey: John Wiley \& Sons, Inc. MR1914507

[2] Bollen, K. A. (1989). Structural Equation Models with Latent Variables. New Jersey: John Wiley \& Sons, Inc. MR0996025

[3] Bollen, K. A., and Curran, P. J. (2006). Latent Curve Models - A Structural Equation Perspective. New Jersey: John Wiley \& Sons, Inc. MR2184502

[4] Cai, J. H., Song, X. Y., Lam, K. H., and IP, H. S. (2011). A mixture of generalized latent variable models for mixed mode and heterogeneous data. Computational Statistics and Data Analysis 55 2889-2907. MR2813054

[5] Caldwell, B. M., and Bradley, R. H. (1984). Home Observation for Measurement of the Environment. Little Rock: University of Arkansas at Little Rock, Center for Child Development and Education.

[6] Celeux, G., Forbes, F., Robert, C. P., and TitteringTON, D. M. (2006). Deviance information criteria for missing data models. Bayesian Analysis 1 651-674. MR2282197

[7] Dempster, A. P., Laird, N. M., and Rubin, D. B. (1977). Maximum likelihood from incomplete data via the EM algorithm. Journal of the Royal Statistical Society, Series B 39 1-38. MR0501537

[8] Duncan, T. E., Duncan, S. C., and Strycker, L. A. (2006). An Introduction to Latent Variable Growth Curve Modeling, 2nd ed. Mahwah, NJ: Lawrence Erlbaum.

[9] Diebolt, J., and Robert, C. P. (1994). Estimation of finite mixture distributions through Bayesian sampling. Journal of the Royal Statistical Society, Series B 56 363-375. MR1281940

[10] Dunn, L. M., and Markwardt, F. C. (1970). Peabody Individual Achievement Test Manual. Circle Pines, NM: American Guidance Services.

[11] Dunson, D. B. (2000). Bayesian latent variable models for clustered mixed outcomes. Journal of the Royal Statistical Society, Series B 62 355-366. MR1749544

[12] Elliott, M. R., Gallo, J. J., Gonger, H. R., and Katz, I. R. (2005). Using a Bayesian latent growth curve model to identify trajectories of positive affect and negative events following myocardial infarction. Biostatistics 6 119-143.

[13] FAHRmeir, L. and RAACH, A. (2007). A Bayesian semiparametric latent variable model for mixed responses. Psychometrika 72327 346. MR2361960

[14] Frühwirth-Schnatter, S. (2001). Markov chain Monte Carlo estimation of classical and dynamic switching and mixture models. Journal of the American Statistical Association 96 194-208. MR1952732

[15] Geman, S., and Geman, D. (1984). Stochastic relaxation, Gibbs distribution, and the Bayesian restoration of images. IEEE Transactions on Pattern Analysis and Machine Intelligence 6 721-741.

[16] Gelman, A. (1996). Inference and monitoring convergence. In: Markov Chain Monte Carlo in Practice, W. R. Gilks, S. Richardson and D. J. Spiegelhalter, eds. London: Chapman and Hall, pp. 131-144. MR1397966

[17] Hastings, W. K. (1970). Monte Carlo sampling methods using Markov chains and their application. Biometrika 57 97-109.

[18] LeE, S. Y. (2007). Structural Equation Modeling - A Bayesian Approach. New York: John Wiley. MR2290938

[19] Lee, S. Y. and Song, X. Y. (2004). Evaluation of Bayesian and maximum likelihood approaches in analyzing structural equation models with small sample sizes. Multivariate Behavioral Research $39653-686$

[20] Lee, S. Y., Song, X. Y., and Poon, W.Y. (2004). Comparison of approaches in estimating interaction and quadratic effects of latent variables. Multivariate Behavioral Research 39 37-67.

[21] Li, Y., and Wang, H. Z. (2010). Bayesian analysis for finite mixture in non-recursive non-linear structural equation models. British Journal of Mathematical and Statistical Psychology 63 361-377 MR2756883
[22] McArdee, J. J., and Bell, R. Q. (2000). Recent trends in modeling longitudinal data by latent growth curve methods. In: Modeling Longitudinal and Multiple-group Data: Practical Issues, Applied Approaches, and Scientific Examples, T. D. Little, K. U. Schnabel, \& J. Baumert, eds. Mahwah, NJ: Lawrence Erlbaum, pp. 69-108.

[23] Meredith, W., and Tisak, J. (1984). On "Tuckerizing" curves. Presented at the annual meeting of the Psychometric Society, Santa Barbara, CA.

[24] Meredith, W., and Tisak, J. (1990). Latent curve analysis. Psychometrika 55(1) 107-122.

[25] Metropolis, N., Rosenbluth, A. W., Rosenbluth, M. N., Teller, A. H., and Teller, E. (1953). Equations of state calculations by fast computing machine. Journal of Chemical Physics 21 1087-1091.

[26] Muthén, B. O. (2001). Latent variable mixture modeling. In New Developments and Techniques in Structural Equation Modeling, G. A. Marcoulides \& R. E. Schumacker, eds. Lawrence Erlbaum Associates, pp. 1-33.

[27] Muthén, B. O., and Curran, J. P. (1997). General longitudinal modeling of individual differences in experimental designs: A latent variable framework for analysis and power estimation. Psychological Methods 2 371-402.

[28] Muthén, B. O., and Shedden, K. (1999). Finite mixture modeling with mixture outcomes using the EM algorithm. Biometrics 55 463-469.

[29] Pan, J. H., Song, X. Y., Lee, S. Y., and Kwok, T. (2008). Longitudinal analysis of quality of life for stroke survivors using latent curve models. Stroke 39 2795-2802.

[30] Roeder, C., and Wasserman, L. (1997). Practical Bayesian density estimation using mixtures of normals. Journal of the American Statistical Association 92 894-902. MR1482121

[31] Sammel, M. D., Ryan, L. M., and Legler, J. M. (1997). Latent variable models for mixed discrete and continuous outcomes. Journal of the Royal Statistical Society, Series B 59 667-678.

[32] Scheines, R., Hoistink, H., and Boomsma, A. (1999). Bayesian estimation and testing of structural equation models. Psychometrika 64 37-52.

[33] Song, X. Y. and Lee, S. Y. (2012). Basic and Advanced Structural Equation Modeling: With Applications in Medical and Behavioural Sciences. John Wiley.

[34] Song, X. Y., and Lu, Z. H. (2010). Semiparametric latent variable models with Bayesian P-splines. Journal of Computational and Graphical Statistics 19 590-608. MR2732494

[35] Song, X. Y., and Lu, Z. H. (2012). Semiparametric transformation models with Bayesian P-splines. Statistics and Computing 22 1085-1098. MR2950087

[36] Song, X. Y., Lu, Z. H., CAI, J. H., and IP, H. S. (2012). A Bayesian modeling approach for generalized semiparametric structural equation models. Psychometrika, accepted.

[37] Song, X. Y., Lee, S. Y., and Hser, Y.I. (2009). Bayesian analysis of multivariate latent curve models with nonlinear longitudinal latent effects. Structural Equation Modeling: A Multidisciplinary Journal 16(2) 245-266. MR2649696

[38] Spiegelhalter, D. J., Best, N. G., Carlin, B. P., and van der Linde, A. (2002). Bayesian measure of model complexity and fit. Journal of the Royal Statistical Society, Series B 64 583-639. MR1979380

[39] Spiegelhalter, D. J., Thomas, A., Best, N. G., and Lunn, D. (2003). WinBUGS User Manual. Version 1.4. Cambridge, England: MRC Biostatistics Unit.

[40] Tanner, M. A., and Wong, W. H. (1987). The calculation of posterier distribution by data augmentation (with discussion). Journal of the American Statistical Association 86 79-86. MR0898357

[41] Willett, J. B., and SAYer, A. G. (1994). Using covariance structure analysis to detect correlates and predictors of change. Psychological Bulletin 116 363-381.

[42] Yung, Y. F. (1997). Finite mixtures in confirmatory factor analysis models. Psychometrika 62 297-330. 
[43] Zill, N. (1985). Behavior Problem Scales Developed From the 1981 Child Health Supplement to the National Health Interview Survey. Washington DC: Child Trends, Inc.

Jun-Hao Pan

Department of Psychology

Sun Yat-Sen University

Guangzhou

China

E-mail address: panjunh@mail.sysu.edu.cn
Xin-Yuan Song

Department of Statistics

The Chinese University of Hong Kong

Hong Kong

China

E-mail address: xysong@sta.cuhk.edu.hk

Edward H. Ip

Department of Biostatistical Sciences

Division of Public Health Sciences

Wake Forest University Health Sciences, NC

United States

E-mail address: eip@wakehealth.edu 